\title{
Existence and Regularity for Boundary Cauchy Problems with Infinite Delay
}

\author{
Jung-Chan Chang \\ Department of Applied Mathematics, I-Shou University, Dashu District, Kaohsiung City 84001, Taiwan \\ Correspondence should be addressed to Jung-Chan Chang; jcchang@isu.edu.tw
}

Received 25 April 2013; Accepted 8 October 2013; Published 20 January 2014

Academic Editor: Fawang Liu

Copyright (C) 2014 Jung-Chan Chang. This is an open access article distributed under the Creative Commons Attribution License, which permits unrestricted use, distribution, and reproduction in any medium, provided the original work is properly cited.

\begin{abstract}
The aim of this work is to investigate a class of boundary Cauchy problems with infinite delay. We give some sufficient conditions ensuring the uniqueness, existence, and regularity of solutions. For illustration, we apply the result to an age dependent population equation, which covers some special cases considered in some recent papers.
\end{abstract}

\section{Introduction}

Consider the following problem:

$$
\begin{gathered}
\frac{\partial}{\partial t} u(t, a)=-\frac{\partial}{\partial a} u(t, a)-\mu(t, a) u(t, a), \quad t \geq 0, a \geq 0, \\
u(t, 0)=f(t), \quad t \geq 0, \\
u(0, a)=\varphi(a), \quad a \geq 0,
\end{gathered}
$$

where $u(t, a)$ represents the density of the population of age $a$ at time $t, \mu$ is the death rate, and $f(t)$ is the number of newborns at time $t$. Such models were introduced by Lotka in 1925 and have been studied by many authors. For a detailed discussion, we refer the reader to $[1,2]$.

The problem (1) can be transformed into the following abstract boundary Cauchy problem:

$$
\begin{gathered}
\frac{d}{d t} u(t)=\mathscr{A} u(t)+\Phi(t) u(t), \quad t \geq 0, \\
L u(t)=f(t) \in \partial \mathscr{X}, \quad t \geq 0, \\
u(0)=x \in \mathscr{X}
\end{gathered}
$$

where $\mathscr{A}$ is an unbounded operator on a Banach space $(\mathscr{X}$, $\|\cdot\|)$ of functions on $[0, \infty)$ with domain $D(\mathscr{A}), u(t)=u(t, \cdot) \epsilon$ $D(\mathscr{A})$ for each $t \geq 0, L: D(\mathscr{A}) \rightarrow \partial \mathscr{X}$ is the operator defined by $L(v(\cdot))=v(0)$ for $v(\cdot) \in D(\mathscr{A})$, and $\partial \mathscr{X}:=\{v(0) ; v(\cdot) \in \mathscr{X}\}$ is a "boundary space." For each $t \geq 0, \Phi(t)$ is a bounded linear operator from $\mathscr{X}$ to $X$.

Equation (2) can be further transformed into a Cauchy problem. To do this, suppose that the domain $D \equiv D(\mathscr{A})$ of $\mathscr{A}$ and $\partial \mathscr{X}$ are Banach spaces such that $D$ is dense and continuously embedded in $\mathscr{X} \cdot \mathscr{A} \in B(D, \mathscr{X})$ and $L \in$ $B(D, \partial \mathscr{X})$. We make the following hypothesis.

(S1) $A:=\left.\mathscr{A}\right|_{\text {ker }(L)}$ generates a $C_{0}$-semigroup $T(\cdot)$ on $\mathscr{X}$ where $\operatorname{ker}(L)$ denotes the kernel of $L$.

(S2) $L$ is a surjection from $D$ to $\partial \mathscr{X} .\left.L\right|_{\mathrm{ker}(\lambda-\mathscr{A})}$ has a continuous inverse for any $\lambda \in \rho(A)$ (the resolvent set of $A$ ).

If assumptions (S1) and (S2) hold, then the operator $P_{\lambda}:=$ $\left(\left.L\right|_{\operatorname{ker}(\lambda-\mathscr{A})}\right)^{-1}$ is continuous from $\partial \mathscr{X}$ to $\mathscr{X}$, and for all $y \in \partial \mathscr{X}$ the operator $P_{\lambda}$ satisfies

$$
(\lambda-\mathscr{A}) P_{\lambda} y=0, \quad L P_{\lambda} y=y .
$$

At least formally, we can rewrite (2) as

$$
\frac{d}{d t} u(t)=A\left(u(t)-P_{\lambda} f(t)\right)+\lambda P_{\lambda} f(t)+\Phi(t) u(t), \quad t \geq 0,
$$

$$
u(0)=x \in \mathscr{X} .
$$


It is easy to see that (4) is a form of the following abstract Cauchy problem:

$$
\begin{gathered}
\frac{d}{d t} u(t)=A\left(u(t)+C G_{1}(t, u(t))\right)+G_{2}(t, u(t)), \quad t \geq 0, \\
u(0)=x \in X
\end{gathered}
$$

where $A$ is the infinitesimal generator of a $C_{0}$-semigroup on a general Banach space $X, C$ is a bounded linear operator satisfying certain conditions, and $G_{1}, G_{2}: \mathbb{R}^{+} \times X \rightarrow X$.

In this way, the problem of solving (1) or (2) is transformed to that of solving (5). Equations of the form like (5) were considered in [3-5]. An important tool used is the multiplicative perturbation which was first studied by Desch and Schappacher [3] in 1989 for $C_{0}$-semigroup. In recent years, this type of perturbations has been further developed and applied by many authors (cf., e.g., Engel and Nagel [6], Piskarëv and Shaw [7]). In this paper, our proof will also be based on an application of the multiplicative perturbation theorem.

Equation (2) has been considered in $[8,9]$ for the cases $f(t)=\int_{0}^{\infty} \beta(t, a) u(t, a) d a$ and $f(t)=\int_{0}^{\infty} \int_{-r}^{\infty} \beta(a, \delta) u(t+$ $\delta, a) d \delta d a$, respectively. Suppose that $\mathscr{B}$ is a linear space of functions from $(-\infty, 0]$ to $\mathscr{X}$. Then these two cases can be viewed as a function from $\mathscr{B}$ to $\mathscr{X}$. That says that $f(t)$ depends on the "history" of $u$. Thus, for such functions $f,(2)$ becomes a retarded Cauchy problem.

The following abstract retarded Cauchy problem has been considered by many authors (see [10-13] and the references therein):

$$
\begin{gathered}
\frac{d}{d t} u(t)=A u(t)+F\left(t, u_{t}\right), \quad t \geq 0, \\
u_{0}=\varphi \in \mathscr{P},
\end{gathered}
$$

where $A$ generates a $C_{0}$-semigroup $T(\cdot)$ on $X, \mathscr{P}$ is a linear space of functions from $(-\infty, 0]$ to $X$ satisfying some axiom which will be described later, $F$ is a function from $[0, \infty) \times \mathscr{P}$ to $X$, and, for a solution function $u: \mathbb{R} \rightarrow X$ and for every $t \geq 0$, the function $u_{t}:(-\infty, 0] \rightarrow X$, defined by

$$
u_{t}(\theta)=u(t+\theta), \quad \text { for } \theta \in(-\infty, 0],
$$

is required to belong to $\mathscr{P}$.

The theory of partial differential equations with infinite delay has attracted widespread attention. In [14-16], the variation-of-constant formula

$$
\begin{gathered}
u(t)=T(t) \varphi(0)+\int_{0}^{t} T(t-s) F\left(s, u_{s}\right) d s, \quad t \geq 0, \\
u_{0}=\varphi \in \mathscr{P}
\end{gathered}
$$

is used to study existence of solutions, regularity, existence of periodic solution, and stability for (6) when the delay is finite. In [10], a similar argument is used to solve (6) when an operator $A$ (not necessarily densely defined) satisfies the Hille-Yosida condition (maybe nondensely defined) and the delay is infinite. For a detailed discussion about infinite delay equations, we refer the reader to [13].
The main purpose of this paper is to consider the following more general boundary Cauchy problem with infinite delay:

$$
\begin{gathered}
\frac{d}{d t} u(t)=\mathscr{A} u(t)+G_{1}\left(t, u_{t}\right), \quad t \geq 0, \\
L u(t)=G_{2}\left(t, u_{t}\right), \quad t \geq 0 \\
u_{0}=\varphi \in \mathscr{P}
\end{gathered}
$$

where $G_{1}$ is a function from $\mathbb{R}^{+} \times \mathscr{P}$ to $\mathscr{X}$ and $G_{2}$ is a function from $\mathbb{R}^{+} \times \mathscr{P}$ to $\partial \mathscr{X} . G_{1}$ and $G_{2}$ may be nonlinear. This abstract boundary delay problem has been studied by Piazzera [8] in some special cases. The case without delay also has been studied in [9]. Similar to the way that (2) is transformed into the form of (5), we can transform (9) into the following generalized retarded abstract Cauchy problem with delay:

$$
\begin{gathered}
\frac{d}{d t} u(t)=A\left(u(t)+C F_{1}\left(t, u_{t}\right)\right)+F_{2}\left(t, u_{t}\right), \quad t \geq 0, \\
u_{0}=\varphi \in \mathscr{P},
\end{gathered}
$$

where $F_{1}, F_{2}$ are functions from $\mathbb{R}^{+} \times \mathscr{P}$ to $X$. It is a generalization of (5) (and hence of (2)) as well as of (6).

In Section 2, we show the uniqueness and existence of solution of (10). It will be solved by using a variationof-constant formula similar to (8). The obtained result (Theorem 7) can be viewed as a partial generalization of $[8,9]$.

Then we apply Theorem 7 in Section 3 to investigate an age dependent population equation for the situation that the birth process depends on the past of the population, as the following system describes:

$$
\begin{gathered}
\frac{\partial}{\partial t} u(t, a)=-\frac{\partial}{\partial a} u(t, a)+f_{1}\left(t, \int_{0}^{\infty} u(t-\tau, \xi) d \xi, a\right), \\
t \geq 0, a \geq 0, \\
u(t, 0)=\int_{0}^{\infty} \int_{-\infty}^{0} G(\theta, a) u(t+\theta, a) d \theta d a+f_{2}(t), \quad t \geq 0, \\
u(t, \theta)=\omega(t, \theta), \quad t \leq 0, \theta \in \mathbb{R}^{+} .
\end{gathered}
$$

This equation contains as particular cases those equations that are considered in the recent papers $[8,9]$.

Finally, we study in Section 4 regularity of mild solutions of (10). The property about equilibrium will be studied. The precise definition of equilibrium will be specified later. In [10], it is shown that the equilibrium of the solution semigroup associated with (6) is locally exponentially stable when its linearized solution around this equilibrium is exponentially bounded. We extend this result to a special case of (10).

\section{Solutions to (10)}

Let $(X,\|\cdot\|)$ be a Banach space. Throughout this paper, $(A$, $D(A))$ is the infinitesimal generator of $C_{0}$-semigroup $T(\cdot)$ on $X$ with domain $D(A)$ and $\|T(t)\| \leq M e^{\omega t}$ for some $M \geq 1, \omega \epsilon$ $\mathbb{R}$, and $t \geq 0$. In this paper, we assume that the phase space 
$\left(\mathscr{P},\|\cdot\|_{\mathscr{P}}\right)$ is a Banach space consisting of some functions from $(-\infty, 0]$ to $X$ and satisfies the following axioms, which were introduced first by Hale and Kato in [17].

(A1) There exist a positive constant $H$ and functions $M_{1}(\cdot)$, $M_{2}(\cdot): \mathbb{R}^{+} \rightarrow \mathbb{R}^{+}$, with $M_{1}$ continuous and $M_{2}$ locally bounded, such that, for any $\sigma \in \mathbb{R}$ and $a \geq 0$, if $x:(-\infty, \sigma+a] \rightarrow X, x_{\sigma} \in \mathscr{P}$ and $x(\cdot)$ is continuous on $[\sigma, \sigma+a]$, then for every $t \in[\sigma, \sigma+a]$ the following conditions hold:

$$
\begin{aligned}
& \text { (i) } x_{t} \in \mathscr{P}, \\
& \text { (ii) }\|x(t)\| \leq H\left\|x_{t}\right\|_{\mathscr{P}}, \\
& \text { (iii) }\left\|x_{t}\right\|_{\mathscr{P}} \leq M_{1}(t-\sigma) \sup _{\sigma \leq s \leq t}\|x(s)\|+M_{2}(t- \\
& \sigma)\left\|x_{\sigma}\right\|_{\mathscr{P}}
\end{aligned}
$$

(A2) For each function $x(\cdot)$ in (A1), $t \mapsto x_{t}$ is a $\mathscr{P}$-valued continuous function on $[\sigma, \sigma+a]$.

The objective of this section is devoted to investigate wellposedness results for the Cauchy problem:

$$
\begin{gathered}
\frac{d}{d t} u(t)=A\left(u(t)+C F_{1}\left(t, u_{t}\right)\right)+F_{2}\left(t, u_{t}\right), \quad t \geq 0, \\
u_{0}=\varphi \in \mathscr{P} .
\end{gathered}
$$

Definition 1. Let $A$ generate a $C_{0}$-semigroup $T(\cdot)$ on $X$. One says that $C \in B(X)$ satisfies condition $(M)$ with respect to $T(\cdot)$ if there is a continuous, nondecreasing function $\sigma_{C}:[0, \infty) \rightarrow$ $[0, \infty)$ with $\sigma_{C}(0)=0$ such that $\int_{0}^{t} T(t-s) C h(s) d s \in D(A)$ and

$$
\left\|A \int_{0}^{t} T(t-s) C h(s) d s\right\| \leq \sigma_{C}(t) \sup _{0 \leq s \leq t}\|h(s)\|,
$$

for each $h \in C([0, t], X)$ and $t \geq 0$.

The following are important examples of operators that satisfy the condition in Definition 1:

(a) $C$ is a bounded linear operator from $X$ to $(D(A)$, $\left.\|\cdot\|_{D(A)}\right)$, the Banach space $D(A)$ endowed with the graph norm $\|x\|_{D(A)}:=\|x\|+\|A x\|$.

(b) $C$ is a bounded linear operator from $X$ to $F(A)$, where $F(A)$ denotes the Favard space of $A$ given by $F(A)=$ $\left\{x \in X ; \limsup _{t \rightarrow 0^{+}}\|T(t) x-x\|<\infty\right\}$ and endowed with the norm $\|x\|_{F(A)}:=\lim \sup _{t \rightarrow 0^{+}}\|T(t) x-x\|+$ $\|x\|$.

These conclusions can be found in $[3,4]$.

In the rest of this paper, we suppose that $C$ satisfies condition $(M)$ with respect to $T(\cdot)$ and the function $\sigma_{C}$ satisfies the corresponding properties. Next, we make the hypotheses about $F_{i}$ for $i=1,2$.

(H1) $F \in C([0, \infty) \times \mathscr{P}, X)$ is continuous and satisfies a Lipschitz condition; that is, there is a constant $L>0$ such that

$$
\left\|F\left(t, \psi_{1}\right)-F\left(t, \psi_{2}\right)\right\| \leq L\left\|\psi_{1}-\psi_{2}\right\|_{\mathscr{P}}
$$

for $\psi_{1}, \psi_{2} \in \mathscr{P}$ and $t \in \mathbb{R}^{+}$.
Definition 2. Let $T>0$. A continuous function $u$ : $(-\infty, T] \rightarrow X$ is called a mild solution of $(12)$ on $[0, T]$ if $u$ satisfies the following conditions:

$$
\begin{aligned}
& \text { (i) } u(t)=T(t) \varphi(0)+A \int_{0}^{t} T(t-s) C F_{1}\left(s, u_{s}\right) d s+\int_{0}^{t} T(t- \\
& s) F_{2}\left(s, u_{s}\right) d s \text { for } t \in[0, T], \\
& \text { (ii) } u(t)=\varphi(t) \text { for } t \in(-\infty, 0] .
\end{aligned}
$$

Definition 3. Let $T>0$. A continuous function $u:(-\infty, T) \rightarrow$ $X$ is called a classical solution of (12) on $[0, T]$ if $u$ satisfies the following conditions:
(i) $u(t)+C F_{1}\left(t, u_{t}\right) \in D(A)$ for $t \in[0, T]$,
(ii) $\left.u\right|_{[0, T]} \in C^{1}([0, T], X)$ and satisfies (12),
(iii) $u(t)=\varphi(t)$ for $t \in(-\infty, 0]$.

First, we show the uniqueness and existence of mild solutions to $(12)$.

Theorem 4. Suppose that $F_{1}$ and $F_{2}$ satisfy hypothesis (H1). Then (12) has a unique mild solution on $[0, T]$ for each $T>0$.

Proof. By assumptions on $F_{1}$ and $F_{2}$, there is a constant $L>0$ independent of $t \in[0, T]$ such that

$$
\sum_{i=1}^{2}\left\|F_{i}\left(t, \phi_{1}\right)-F_{i}\left(t, \phi_{2}\right)\right\| \leq L\left\|\phi_{1}-\phi_{2}\right\|_{\mathscr{P}}
$$

for $t \in[0, T]$ and $\phi_{1}, \phi_{2} \in \mathscr{P}$. Moreover, we define the following real number:

$$
M_{T}^{\prime \prime}=L M_{T}^{\prime} M e^{|\omega| T},
$$

where $M_{T}^{\prime}=\max \left\{M_{1}^{\prime}, M_{2}^{\prime}\right\}, M_{i}^{\prime}=\sup _{0 \leq s \leq T} M_{i}(s), i=1,2$, and $M_{1}(\cdot)$ and $M_{2}(\cdot)$ are the functions defined in hypothesis (A1). Note that $M_{T}^{\prime \prime}$ is independent of $t \in[0, T]$. Let $[0, r] \subset$ $[0, T]$ and let

$$
Z(r):=\left\{y:(-\infty, r] \longrightarrow X ; y_{0} \in \mathscr{P},\left.y\right|_{[0, r]} \in C([0, r], X)\right\}
$$

be a Banach space equipped with the norm

$$
\|y\|_{Z_{r}}:=\left\|y_{0}\right\|_{\mathscr{P}}+\|y\|_{C([0, r], X)} .
$$

Let

$$
Z_{\varphi}(r):=\left\{y \in Z(r) ; y_{0}=\varphi\right\} .
$$

Then $Z_{\varphi}(r)$ is a closed subset of $Z(r)$.

Note that it follows from (A2) and (H1) that $F_{i}\left(s, v_{s}\right)$, $i=1,2$, are continuous in $s$ on $[0, r]$. Then, since $C$, satisfies condition $(M)$, we have $A \int_{0}^{t} T(t-s) C F_{1}\left(s, v_{s}\right) d s \in C([0, r]$, $X$ ) (see the proof of Theorem 2.2 in [7]). Thus we can define $S_{r}^{\varphi}: Z_{\varphi}(r) \rightarrow Z_{\varphi}(r)$ by

$$
\begin{aligned}
& \left(S_{r}^{\varphi} v\right)(t) \\
& :=\left\{\begin{array}{cc}
T(t) \varphi(0)+A \int_{0}^{t} T(t-s) C F_{1}\left(s, v_{s}\right) d s \\
+\int_{0}^{t} T(t-s) F_{2}\left(s, v_{s}\right) d s, & t \in[0, r], \\
\varphi(t), & t \in(-\infty, 0] .
\end{array}\right.
\end{aligned}
$$


Note that the closed set $Z_{\varphi}(r)$ and the operator $S_{r}^{\varphi}$ are dependent on $r$ and $\varphi$. From the definition of $S_{r}^{\varphi}$, one can see that the fixed point of $S_{r}^{\varphi}$ is a mild solution of (12) on $[0, r]$. Furthermore, if $r=T$ and $S_{T}^{\varphi}$ has a unique fixed point, then the fixed point is the unique solution to (12) from the definition of $S_{T}^{\varphi}$ and the proof is completed. So, it is sufficient to show that $S_{T}^{\varphi}$ has a unique fixed point in $Z_{\varphi}(T)$. The unique fixed point will be found step by step. First, we show that there is an $r \in(0, T]$ such that $S_{r}^{\varphi}$ has a unique fixed point. This fact will be shown by finding an $r \in(0, T]$ such that $S_{r}^{\varphi}$ is a contraction. Suppose that $v_{1}, v_{2} \in Z_{\varphi}(r)$. For $t \in[0, r]$, by the definition of $S_{r}^{\varphi}$, assumption of $C$ and hypotheses (A1) and (A2), it follows that

$$
\begin{aligned}
& \left\|\left(S_{r}^{\varphi} v_{1}-S_{r}^{\varphi} v_{2}\right)(t)\right\| \\
& \leq\left\|A \int_{0}^{t} T(t-s) C\left(F_{1}\left(s,\left(v_{1}\right)_{s}\right)-F_{1}\left(s,\left(v_{2}\right)_{s}\right)\right) d s\right\| \\
& \quad+\left\|\int_{0}^{t} T(t-s)\left(F_{2}\left(s,\left(v_{1}\right)_{s}\right)-F_{2}\left(s,\left(v_{2}\right)_{s}\right)\right) d s\right\| \\
& \leq \sigma_{C}(t) \sup _{0 \leq s \leq t}\left\|F_{1}\left(s,\left(v_{1}\right)_{s}\right)-F_{1}\left(s,\left(v_{2}\right)_{s}\right)\right\| \\
& \quad+M t^{|\omega| t} \int_{0}^{t}\left\|F_{2}\left(s,\left(v_{1}\right)_{s}\right)-F_{2}\left(s,\left(v_{2}\right)_{s}\right)\right\| d s \\
& \leq \sigma_{C}(t) L M_{T}^{\prime}\left\|v_{1}-v_{2}\right\|_{Z_{r}}+M e^{|\omega| t} L M_{T}^{\prime} t\left\|v_{1}-v_{2}\right\|_{Z_{r}} \\
& \leq\left(\sigma_{C}(r)+r\right) M_{T}^{\prime \prime}\left\|v_{1}-v_{2}\right\|_{Z_{r}} .
\end{aligned}
$$

So, by the assumption on $\sigma_{C}$, there exist $N \in \mathbb{N}$ and $r \in(0, T]$ such that $N r=T$ and $\left(\sigma_{C}(t)+t\right) M_{T}^{\prime \prime}<1$ for each $t \in[0, r]$. On the other hand, $\left(S_{r}^{\varphi} v_{1}-S_{r}^{\varphi} v_{2}\right)(t)=\varphi(t)-\varphi(t)=0$ for all $t \leq 0$. It follows that $S_{r}^{\varphi}$ is a contraction on $Z_{\varphi}(r)$. Hence $S_{r}^{\varphi}$ has a unique fixed point $u^{1} \in Z_{\varphi}(r)$ by the contraction mapping principle.

If $r=T$, then the proof is completed. Next, if $2 r \leq T$, then the previous argument will be repeated. Let us define the function $\varsigma:(-\infty, 0]$ by

$$
\varsigma(t)=\left(u^{1}\right)_{r}(t)= \begin{cases}u^{1}(t+r), & t \in[-r, 0], \\ \varphi(t+r), & t \in(-\infty,-r] .\end{cases}
$$

Since $u^{1} \in Z_{\varphi}(r)$ implies $u^{1}:(-\infty, r] \rightarrow X$ with $u^{1} \in$ $C([0, r], X)$ and $\left(u^{1}\right)_{0}=\varphi$, it follows from the hypotheses (A1)(i) that $\varsigma=\left(u^{1}\right)_{r} \in \mathscr{P}$.

Now, we can define the closed set $Z_{\zeta}(r)$ of $Z(r)$ and define the operator from $Z_{\varsigma}(r)$ to $Z_{\varsigma}(r)$ by

$$
\begin{aligned}
& \left(S_{r}^{\varsigma} v\right)(t) \\
& \quad=\left\{\begin{array}{cc}
T(t) \varsigma(0)+A \int_{0}^{t} T(t-s) C F_{1}\left(s+r, v_{s}\right) d s \\
+\int_{0}^{t} T(t-s) F_{2}\left(s+r, v_{s}\right) d s, & t \in[0, r], \\
\varsigma(t), & t \in(-\infty, 0],
\end{array}\right.
\end{aligned}
$$

for each $v \in Z_{\zeta}(r)$ and $t \in[0, r]$. Repeating the previous argument, $S_{r}^{\varsigma}$ has a unique fixed point $u^{2}$ in $Z_{\varsigma}(r)$. Define $w$ : $(-\infty, 2 r]$ by

$$
w(t)= \begin{cases}\varphi(t), & -\infty \leq t \leq 0 \\ u^{1}(t), & 0 \leq t \leq r, \\ u^{2}(h), & r \leq r+h=t \leq 2 r .\end{cases}
$$

Then, we show that $w$ is a fixed point of $S_{2 r}^{\varphi}$ on $Z_{\varphi}(2 r)$. If $0 \leq$ $t \leq r$, then $w(t)=u^{1}(t)=S_{r}^{\varphi}\left(u^{1}\right)(t)$, so that it follows from (20) that

$$
\begin{aligned}
w(t)= & T(t) \varphi(0)+A \int_{0}^{t} T(t-s) C F_{1}\left(s, w_{s}\right) d s \\
& +\int_{0}^{t} T(t-s) F_{2}\left(s, w_{s}\right) d s .
\end{aligned}
$$

In particular, for $t=r$, it becomes

$$
\begin{aligned}
\varsigma(0)= & u^{1}(r)=w(r) \\
= & T(r) \varphi(0)+A \int_{0}^{r} T(r-s) C F_{1}\left(s, w_{s}\right) d s \\
& +\int_{0}^{r} T(r-s) F_{2}\left(s, w_{s}\right) d s .
\end{aligned}
$$

If $r \leq t \leq 2 r$, let $t=r+h$; then by (26) one has

$$
\begin{aligned}
w(t)= & u^{2}(h)=\left(S_{r}^{\varsigma} u^{2}\right)(h) \\
= & T(h) \varsigma(0)+A \int_{0}^{h} T(h-s) C F_{1}\left(s+r,\left(u^{2}\right)_{s}\right) d s \\
& +\int_{0}^{h} T(h-s) F_{2}\left(r+s,\left(u^{2}\right)_{s}\right) d s \\
= & T(r+h) \varphi(0)+A \int_{0}^{r} T(r+h-s) C F_{1}\left(s, w_{s}\right) d s \\
& +\int_{0}^{r} T(r+h-s) F_{2}\left(s, w_{s}\right) d s \\
& +A \int_{r}^{h+r} T(h+r-s) C F_{1}\left(s, w_{s}\right) d s \\
& +\int_{r}^{h+r} T(r+h-s) F_{2}\left(s,(w)_{s}\right) d s \\
= & T(t) \varphi(0)+A \int_{0}^{t} T(t-s) C F_{1}\left(s, w_{s}\right) d s \\
& +\int_{0}^{t} T(t-s) F_{2}\left(s, w_{s}\right) d s .
\end{aligned}
$$

Hence $w$ is a fixed point of $S_{2 r}^{\varphi}$ in $Z_{\varphi}(2 r)$. Since $u^{1}$ and $u^{2}$ are the unique points in $Z_{\varphi}(r)$ and $Z_{\zeta}(r)$, respectively, it follows that $w$ is the unique fixed point in $Z_{\varphi}(2 r)$. This argument can be repeated until $N r=T$. At the end, we can find the unique fixed point of $S_{T}^{\varphi}$ on $Z_{\varphi}(T)$. 
Next, we want to give a sufficient condition for the existence of classical solution to (12). To do this, we need the differentiability of mild solutions. We give the following more restrictive conditions.

(B) If $\left(\phi_{n}\right)$ is a Cauchy sequence in $\mathscr{P}$ and if $\left(\phi_{n}\right)$ converges compactly to $\phi$ on $(-\infty, 0]$ (i.e., for each compact subset $E$ of $\mathbb{R}^{-},\left.f_{n}\right|_{E}$ is convergent uniformly to $\left.\phi\right|_{E}$ ), then $\phi \in \mathscr{P}$ and $\left\|\phi_{n}-\phi\right\|_{\mathscr{P}} \rightarrow 0$, as $n \rightarrow \infty$.

(C) For a sequence $\left(\phi_{n}\right)$ in $\mathscr{P}$, if $\left\|\phi_{n}\right\|_{\mathscr{P}} \rightarrow 0$ as $n \rightarrow \infty$, then $\left\|\phi_{n}(\theta)\right\| \rightarrow 0$, as $n \rightarrow \infty$, for each $\theta \in(-\infty, 0]$.

(H2) $F: \mathbb{R} \times \mathscr{P} \rightarrow X$ is continuously differentiable and the derivatives $D_{1} F, D_{2} F$ satisfy the following Lipschitz conditions: there is a constant $L>0$ such that

$$
\begin{gathered}
\left\|D_{1} F\left(t, \psi_{1}\right)-D_{1} F\left(t, \psi_{2}\right)\right\| \leq L\left\|\psi_{1}-\psi_{2}\right\|_{\mathscr{P}}, \\
\left\|D_{2} F\left(t, \psi_{1}\right)-D_{2} F\left(t, \psi_{2}\right)\right\| \leq L\left\|\psi_{1}-\psi_{2}\right\|_{\mathscr{P}},
\end{gathered}
$$

for $t \in[0, \infty)$ and $\psi_{1}, \psi_{2} \in \mathscr{P}$, where $D_{i}$ denotes the derivative with respect to the $i$ th variable.

The following lemmas are needed.

Lemma 5 (see [13]). Let $\mathscr{P}$ satisfy axiom $(B)$ and let $f$ : $[0, a] \rightarrow \mathscr{P}, a>0$, be a continuous function such that $f(t)(\theta)$ is continuous for $(t, \theta) \in[0, a] \times(-\infty, 0]$. Then

$$
\left[\int_{0}^{a} f(t) d t\right](\theta)=\int_{0}^{a} f(t)(\theta) d t
$$

for $\theta \in(-\infty, 0]$.

Lemma 6 (see [18]). Let $\mathscr{P}$ satisfy axiom $(C)$ and let $f$ : $[0, a] \rightarrow \mathscr{P}, a>0$, be a continuous function. Then for all $\theta \in(-\infty, a]$, the function $f(\cdot)(\theta)$ is continuous and

$$
\left[\int_{0}^{a} f(t) d t\right](\theta)=\int_{0}^{a} f(t)(\theta) d t
$$

for $\theta \in(-\infty, 0]$.

Theorem 7. Let $\mathscr{P}$ satisfy axiom (B) or (C). Assume that $F_{1}$ and $F_{2}$ satisfy assumptions (H1) and (H2). In addition, assume that $\varphi \in \mathscr{P}$ is continuously differentiable with $\varphi^{\prime} \in \mathscr{P}, \varphi(0)+$ $C F_{1}(0, \varphi) \in D(A)$, and $\varphi^{\prime}(0)=A\left(\varphi(0)+C F_{1}(0, \varphi)\right)+F_{2}(0, \varphi)$. If $u(\cdot, \varphi)$ is the unique mild solution of $(12)$ on $[0, T]$, then $u$ is continuously differentiable on $[0, T]$. Furthermore, $u(\cdot, \varphi)$ is a classical solution of (12) on $[0, T]$.

Proof. Consider the following equation:

$$
\begin{gathered}
y(t)=T(t)\left[A\left(\varphi(0)+C F_{1}(0, \varphi)\right)+F_{2}(0, \varphi)\right] \\
+A \int_{0}^{t} T(t-s) C\left(D_{1} F_{1}\left(s, u_{s}\right)+D_{2} F_{1}\left(s, u_{s}\right) y_{s}\right) d s \\
+\int_{0}^{t} T(t-s)\left(D_{1} F_{2}\left(s, u_{s}\right)+D_{2} F_{2}\left(s, u_{s}\right) y_{s}\right) d s, \\
t \in[0, T], \\
y_{0}=\varphi^{\prime} \in \mathscr{P} .
\end{gathered}
$$

A similar argument as in the proof of Theorem 4 shows that there is a unique solution $y$ to $(31)$ on $[0, T]$. Define the function $z$ by

$$
z(t)= \begin{cases}\varphi(0)+\int_{0}^{t} y(s) d s, & t \in[0, T], \\ \varphi(t), & t \in(-\infty, 0] .\end{cases}
$$

We first show that if there is an $r>0$ such that $u=z$ on $[0, r]$, then $u$ is a classical solution of $(12)$ on $[0, r]$. In fact, in this case, $u$ is a differentiable mild solution. Denote $G_{i}(s)=$ $F_{i}\left(s, u_{s}\right)$ for $i=1,2$. It is easy to see that $G_{i}$ is continuously differentiable. Using integration by parts, we can write

$$
\begin{aligned}
A \int_{0}^{t} T(t-s) C G_{1}(s) d s \\
=-\left.A \int_{0}^{t-s} T(r) C G_{1}(s) d r\right|_{s=0} ^{s=t} \\
\quad+A \int_{0}^{t} \int_{0}^{t-s} T(r) C G_{1}^{\prime}(s) d r d s \\
=\int_{0}^{t} T(t-s) C G_{1}^{\prime}(s) d s-C G_{1}(t)+T(t) C G_{1}(0) .
\end{aligned}
$$

So, from the definition of mild solution, it follows that $u(t)+$ $C G_{1}(t)=T(t)\left(\varphi(0)+C G_{1}(0)\right)+\int_{0}^{t} T(t-s) C G_{1}^{\prime}(s) d s+\int_{0}^{t} T(t-$ s) $G_{2}(s) d s$. Furthermore, by the assumption, $\varphi(0)+C G_{1}(0) \epsilon$ $D(A)$, and Definition 1, we see that $u(t)+C G_{1}(t) \in D(A)$ for each $t \geq 0$. Hence,

$$
\begin{aligned}
& A\left(u(t)+C G_{1}(t)\right) \\
& =A\left[T(t)\left(\varphi(0)+C G_{1}(0)\right)+\int_{0}^{t} T(t-s) C G_{1}^{\prime}(s) d s\right. \\
& \left.\quad+\int_{0}^{t} T(t-s) G_{2}(s) d s\right] \\
& =T(t) A\left(\varphi(0)+C G_{1}(0)\right)+A \int_{0}^{t} T(t-s) C G_{1}^{\prime}(s) d s \\
& \quad+A \int_{0}^{t} T(t-s) G_{2}(s) d s \\
& =T(t) A\left(\varphi(0)+C G_{1}(0)\right)+A \int_{0}^{t} T(t-s) C G_{1}^{\prime}(s) d s \\
& \quad+\int_{0}^{t} T(t-s) G_{2}^{\prime}(s) d s+T(t) G_{2}(0)-G_{2}(t) .
\end{aligned}
$$

On the other hand, we see that

$$
\begin{aligned}
u(t)= & A\left[\int_{0}^{t} T(r) \varphi(0) d r+\int_{0}^{t} T(t-s) C G_{1}(s) d s\right] \\
& +\varphi(0)+\int_{0}^{t} T(t-s) G_{2}(s) d s .
\end{aligned}
$$


So,

$$
\begin{aligned}
u(t) & -\left[\varphi(0)+\int_{0}^{t} T(t-s) G_{2}(s) d s\right] \\
& =A\left[\int_{0}^{t} T(r) \varphi(0) d r+\int_{0}^{t} T(t-s) C G_{1}(s) d s\right] .
\end{aligned}
$$

Differentiating both sides, we obtain

$$
\begin{aligned}
& u^{\prime}(t)-T(t) G_{2}(0)-\int_{0}^{t} T(t-s) G_{2}^{\prime}(s) d s \\
& \quad=T(t) A\left(\varphi(0)+G_{1}(0)\right)+A \int_{0}^{t} T(t-s) C G_{1}^{\prime}(s) d s .
\end{aligned}
$$

Finally, comparing $(*)$ with $(* *)$, we see that $u$ is a classical solution on $[0, r]$.

Next, we show that there does exist an $r>0$ such that $u=z$ on $[0, r]$. Recall the integrated semigroup $S(\cdot)$ generated by $A$; that is, $S(t) x=\int_{0}^{t} T(s) x d s$ for each $x \in X$. One can obtain that

$$
\begin{aligned}
& \int_{0}^{t} T(s)\left[A\left(\varphi(0)+C F_{1}(0, \varphi)\right)+F_{2}(0, \varphi)\right] d s \\
& \quad=\int_{0}^{t} T(s) \varphi^{\prime}(0) d s \\
& \quad=S(t) \varphi^{\prime}(0), \\
& \int_{0}^{t} \int_{0}^{s} T(s-r) C^{2-i}\left(D_{1} F_{i}\left(r, u_{r}\right)+D_{2} F_{i}\left(r, u_{r}\right) y_{r}\right) d r d s \\
& \quad=\int_{0}^{t} \int_{r}^{t} T(s-r) C^{2-i}\left(D_{1} F_{i}\left(r, u_{r}\right)+D_{2} F_{i}\left(r, u_{r}\right) y_{r}\right) d s d r \\
& =\int_{0}^{t} S(t-r) C^{2-i}\left(D_{1} F_{i}\left(r, u_{r}\right)+D_{2} F_{i}\left(r, u_{r}\right) y_{r}\right) d r,
\end{aligned}
$$

for $i=1,2$. Here $C^{0}$ denotes the identity map. Therefore, by the closedness of $A$ and the assumption on $C, z$ becomes

$$
\begin{aligned}
& z(t)=\varphi(0)+S(t) \varphi^{\prime}(0) \\
& +\sum_{i=1}^{2} A^{2-i} \int_{0}^{t} S(t-s) C^{2-i} \\
& \quad \times\left(D_{1} F\left(s, u_{s}\right)+D_{2} F\left(s, u_{s}\right) y_{s}\right) d s,
\end{aligned}
$$

for $t \in[0, T]$, where $A^{0}$ denotes the identity map. By Lemma 5 or Lemma 6, we obtain

$$
z_{t}=\varphi+\int_{0}^{t} y_{s} d s \quad \text { for } t \in[0, T] .
$$

By the elementary properties of $S(\cdot)$,

$$
\begin{aligned}
S(t) \varphi^{\prime}(0)= & T(t)\left(\varphi(0)+C F_{1}(0, \varphi)\right) \\
& -\left(\varphi(0)+C F_{1}(0, \varphi)\right)+S(t) F_{2}(0, \varphi) \\
= & T(t) \varphi(0)-\varphi(0)+A S(t) C F_{1}(0, \varphi) \\
& +S(t) F_{2}(0, \varphi) \\
= & T(t) \varphi(0)-\varphi(0)+\sum_{i=1}^{2} A^{2-i} S(t) C^{2-i} F_{i}(0, \varphi),
\end{aligned}
$$

for $t \in[0, T]$. Moreover, using integration by parts and simple computation, one can derive that

$$
\begin{aligned}
A^{2-i} \int_{0}^{t} T(t-s) C^{2-i} F_{i}\left(s, z_{s}\right) d s \\
=A^{2-i} S(t) C^{2-i} F_{i}(0, \varphi) \\
\quad+A^{2-i} \int_{0}^{t} S(t-s) C^{2-i}\left(D_{1} F_{i}\left(s, z_{s}\right)+D_{2} F_{i}\left(s, z_{s}\right) y_{s}\right) d s,
\end{aligned}
$$

for $t \in[0, T]$ and $i=1,2$. Consequently, by (36)-(40), $z$ satisfies

$$
\begin{aligned}
z(t)= & T(t) \varphi(0)+\sum_{i=1}^{2} A^{2-i} S(t) C^{2-i} F_{i}(0, \varphi) \\
+ & \sum_{i=1}^{2} A^{2-i}\left[\int_{0}^{t} S(t-s) C^{2-i}\right. \\
& \left.\times\left(D_{1} F_{i}\left(s, u_{s}\right)+D_{2} F_{i}\left(s, u_{s}\right) y_{s}\right) d s\right] \\
= & T(t) \varphi(0) \quad \\
+\sum_{i=1}^{2} A^{2-i}[ & -\int_{0}^{t} S(t-s) C^{2-i} \\
& \times\left(D_{1} F_{i}\left(s, z_{s}\right)+D_{2} F_{i}\left(s, z_{s}\right) y_{s}\right) d s \\
& +\int_{0}^{t} T(t-s) C^{2-i} F_{i}\left(s, z_{s}\right) d s \\
& +\int_{0}^{t} S(t-s) C^{2-i} \\
& \left.\times\left(D_{1} F_{i}\left(s, u_{s}\right)+D_{2} F_{i}\left(s, u_{s}\right) y_{s}\right) d s\right]
\end{aligned}
$$


for $t \in[0, T]$. On the other hand, by hypotheses $(\mathrm{H} 1)$ and (H2), there exists $L>0$ such that

$$
\begin{aligned}
& \sum_{i=1}^{2}\left\|F_{i}\left(t, \phi_{1}\right)-F_{i}\left(t, \phi_{2}\right)\right\|+\sum_{i=1}^{2}\left\|D_{i} F_{1}\left(t, \phi_{1}\right)-D_{i} F_{1}\left(t, \phi_{2}\right)\right\| \\
& +\sum_{i=1}^{2}\left\|D_{i} F_{2}\left(t, \phi_{1}\right)-D_{i} F_{2}\left(t, \phi_{2}\right)\right\| \leq L\left\|\phi_{1}-\phi_{2}\right\|_{\mathscr{P}}
\end{aligned}
$$

for $\phi_{1}, \phi_{2} \in \mathscr{P}$. Moreover, we define the following real number:

$$
M_{T}^{\prime \prime}=L M_{T}^{\prime} M e^{|\omega| T}
$$

where $M_{T}^{\prime}=\sup _{0 \leq s \leq T} M_{2}(s)$ and $M_{2}(\cdot)$ is the function defined in hypothesis (A1). Therefore, by (41) and definition of $u$, one can obtain that

$$
\begin{aligned}
& \|u(t)-z(t)\| \\
& \leq \sum_{i=1}^{2}\left[\left\|A^{2-i} \int_{0}^{t} T(t-s) C^{2-i}\left(F_{i}\left(s, u_{s}\right)-F_{i}\left(s, z_{s}\right)\right) d s\right\|\right. \\
& +\| A^{2-i} \int_{0}^{t} S(t-s) C^{2-i}\left(D_{1} F_{i}\left(s, u_{s}\right)\right. \\
& \left.\quad \| A^{2-i} \int_{0}^{t} S(t-s) C^{2-i}\left(s, z_{s}\right)\right) d s \| \\
& \left.\quad \times\left(D_{2} F_{i}\left(s, u_{s}\right) y_{s}-D_{2} F_{i}\left(s, z_{s}\right) y_{s}\right) d s \|\right] .
\end{aligned}
$$

For $i=1$, by (13), we have

$$
\begin{aligned}
& \left\|A \int_{0}^{t} T(t-s) C\left(F_{1}\left(s, u_{s}\right)-F_{1}\left(s, z_{s}\right)\right) d s\right\| \\
& \quad \leq \sigma_{C}(t) \sup _{0 \leq s \leq t}\left\|\left(F_{1}\left(s, u_{s}\right)-F_{1}\left(s, z_{s}\right)\right)\right\|, \\
& \left\|A \int_{0}^{t} S(t-s) C\left(D_{1} F_{1}\left(s, u_{s}\right)-D_{1} F_{1}\left(s, z_{s}\right)\right) d s\right\| \\
& \quad=\left\|\int_{0}^{t} A \int_{0}^{t-s} T(r) C\left(D_{1} F_{1}\left(s, u_{s}\right)-D_{1} F_{1}\left(s, z_{s}\right)\right) d r d s\right\| \\
& \quad \leq \int_{0}^{t}\|T(t-s)-I\|\left\|C\left(D_{1} F_{1}\left(s, u_{s}\right)-D_{1} F_{1}\left(s, z_{s}\right)\right)\right\| d s \\
& \quad \leq 2 t M e^{|\omega| T} \sup _{0 \leq s \leq t}\left\|C\left(D_{1} F_{1}\left(s, u_{s}\right)-D_{1} F_{1}\left(s, z_{s}\right)\right)\right\|,
\end{aligned}
$$

and similarly

$$
\begin{aligned}
& \left\|A \int_{0}^{t} S(t-s) C\left(D_{2} F_{1}\left(s, u_{s}\right) y_{s}-D_{2} F_{1}\left(s, z_{s}\right) y_{s}\right) d s\right\| \\
& \quad \leq 2 t M e^{|\omega| T} \sup _{0 \leq s \leq t}\left\|C\left(D_{2} F_{1}\left(s, u_{s}\right) y_{s}-D_{2} F_{1}\left(s, z_{s}\right) y_{s}\right)\right\| .
\end{aligned}
$$

Therefore

$$
\begin{aligned}
\left\|A \int_{0}^{t} T(t-s) C\left(F_{1}\left(s, u_{s}\right)-F_{1}\left(s, z_{s}\right)\right) d s\right\| \\
\quad+\left\|A \int_{0}^{t} S(t-s) C\left(D_{1} F_{1}\left(s, u_{s}\right)-D_{1} F_{1}\left(s, z_{s}\right)\right) d s\right\| \\
\quad+\left\|A \int_{0}^{t} S(t-s) C\left(D_{2} F_{1}\left(s, u_{s}\right) y_{s}-D_{2} F_{1}\left(s, z_{s}\right) y_{s}\right) d s\right\| \\
\leq \sigma_{C}(t) M_{T}^{\prime \prime} \sup _{0 \leq s \leq t}\|u(s)-z(s)\| \\
\quad+2 t M_{T}^{\prime \prime}\|C\|\left(1+\sup _{0 \leq s \leq T}\left\|y_{s}\right\|_{\mathscr{P}}\right) \sup _{0 \leq s \leq t}\|u(s)-z(s)\| \\
=M_{T}^{\prime \prime}\left[\sigma_{C}(t)+2 t\|C\|\left(1+\sup _{0 \leq s \leq T}\left\|y_{s}\right\|_{\mathscr{P}}\right)\right] \\
\quad \times \sup _{0 \leq s \leq t}\|u(s)-z(s)\| \\
=K \sup _{0 \leq s \leq t}\|u(s)-z(s)\|,
\end{aligned}
$$

where

$$
K:=M_{T}^{\prime \prime}\left[\sigma_{C}(r)+2 r\|C\|\left(1+\max _{0 \leq s \leq T}\|y\|_{\mathscr{P}}\right)\right] .
$$

By the assumption on $\sigma_{C}$, we can choose $N \in \mathbb{N}$ and $r>0$ such that $N r=T$ and $K<1$.

For $i=2$,

$$
\begin{aligned}
& \left\|\int_{0}^{t} T(t-s)\left(F_{2}\left(s, u_{s}\right)-F_{2}\left(s, z_{s}\right)\right) d s\right\| \\
& \quad+\left\|\int_{0}^{t} S(t-s)\left(D_{1} F_{2}\left(s, u_{s}\right)-D_{1} F_{2}\left(s, z_{s}\right)\right) d s\right\| \\
& \quad+\left\|\int_{0}^{t} S(t-s)\left(D_{2} F_{2}\left(s, u_{s}\right) y_{s}-D_{2} F_{2}\left(s, z_{s}\right) y_{s}\right) d s\right\| \\
& \leq\left(M_{T}^{\prime \prime}+M_{T}^{\prime \prime} T+M_{T}^{\prime \prime} T \times \max _{0 \leq s \leq T}\left\|y_{s}\right\|_{\mathscr{P}}\right) \\
& \quad \times \int_{0}^{t} \sup _{0 \leq \zeta \leq s}\|u(\zeta)-z(\zeta)\| d s .
\end{aligned}
$$


Consequently, from (44), (47), and (49), one can derive that

$$
\begin{aligned}
& \sup _{0 \leq \zeta \leq t}\|u(\zeta)-z(\zeta)\| \\
& \leq \frac{1}{1-K}\left(M_{T}^{\prime \prime}+M_{T}^{\prime \prime} T+M_{T}^{\prime \prime} T \times \max _{0 \leq s \leq T}\left\|y_{s}\right\|_{\mathscr{P}}\right) \\
& \quad \times \int_{0}^{t} \sup _{0 \leq \zeta \leq s}\|u(\zeta)-z(\zeta)\| d s,
\end{aligned}
$$

for $0 \leq t \leq r$. By a standard argument and using Gronwall's inequality, we get $u=z$ on $[0, r]$. So, we have derived that $u$ is continuously differentiable on $[0, r]$, and hence $u$ is a classical solution of $(12)$ on $[0, r]$.

If $r=T$, then the proof is completed. If $r \leq t=r+h \leq 2 r$, from the definition (Definition 2(i)) of $u$ we see that

$$
\begin{aligned}
u(r+ & h) \\
= & T(r+h) \varphi(0)+A \int_{0}^{r+h} T(r+h-s) C F_{1}\left(s, u_{s}\right) d s \\
& +\int_{0}^{r+h} T(r+h-s) F_{2}\left(s, u_{s}\right) d s \\
= & T(r+h) \varphi(0)+A \int_{0}^{r} T(r+h-s) C F_{1}\left(s, u_{s}\right) d s \\
& +A \int_{r}^{r+h} T(r+h-s) C F_{1}\left(s, u_{s}\right) d s \\
& +\int_{0}^{r} T(r+h-s) F_{2}\left(s, u_{s}\right) d s \\
& +\int_{r}^{r+h} T(h+h-s) F_{2}\left(s, u_{s}\right) d s \\
& +\int_{r}^{r+h} T(r+h-s) F_{2}\left(s, u_{s}\right) d s \\
= & T(h)\left[(r)+A \int_{0}^{h} T(h-s) C\left(F_{1}\left(r+s, u_{r+s}\right)\right) d s\right. \\
& \left.+\int_{0}^{r} T(r-s) F_{2}\left(s, u_{s}\right) d s\right] \\
& +(0)+A \int_{0}^{r} T(r-s) C F_{1}\left(s, u_{s}\right) d s
\end{aligned}
$$

It follows that $u(r+\cdot)$ is a mild solution of

$$
\begin{gathered}
\frac{d}{d t} x(t)=A\left(x(t)+C F_{1}\left(r+t, x_{t}\right)\right)+F_{2}\left(r+t, x_{t}\right), \\
t \in[0, r], \\
x_{0}=u_{r} \in \mathscr{P} .
\end{gathered}
$$

Repeating the previous argument, we can show that $u(r+\cdot)$ is differentiable on $[0, r]$. Hence, $u$ is a classical solution of (12) on $[0,2 r]$. This argument can be repeated until $N r=T$. At the end, one can show that $u$ is a classical solution of (12) on $[0, T]$.

\section{Application to Age Dependent Population Equations}

In this section, the results in the previous section will be applied to age dependent population equations.

Theorem 8 (see [13]). Let $\left(X,\|\cdot\|_{X}\right)$ be a Banach space and let $\gamma>0$ be a fixed number. Suppose that $\mathscr{P}$ denotes the space $\mathscr{P}:=\left\{\phi:(-\infty, 0] \rightarrow X ; \lim _{\theta \rightarrow-\infty} e^{\gamma \theta} \phi(\theta) \in X\right\}$ endowed with the norm $\|\phi\|_{\mathscr{P}}:=\sup _{0 \geq \theta} e^{\gamma \theta}\|\phi(\theta)\|_{X}$; then $\mathscr{P}$ satisfies assumptions (A1), (A2), (B), and (C).

Let $X=L^{1}\left(\mathbb{R}^{+}, \mathbb{R}\right)$ with $L^{1}$ norm $\|\cdot\|$, and let $\mathscr{P}$ be the phase space $\left\{\phi:(-\infty, 0] \rightarrow X ; \lim _{\theta \rightarrow-\infty} e^{\gamma \theta} \phi(\theta) \in X\right\}$ endowed with the norm $\|\phi\|_{\mathscr{P}}:=\sup _{0 \geq \theta} e^{\gamma \theta}\|\phi(\theta)\|$ for a fixed $\gamma>0$. Let us consider the following system:

$$
\begin{gathered}
\frac{\partial}{\partial t} u(t, a)=-\frac{\partial}{\partial a} u(t, a)+f_{1}\left(t, \int_{0}^{\infty} u(t-\tau, \xi) d \xi, a\right) \\
t \geq 0, a \geq 0, \\
u(t, 0)=\int_{0}^{\infty} \int_{-\infty}^{0} G(\theta, a) u(t+\theta, a) d \theta d a+f_{2}(t), \quad t \geq 0 \\
u(t, \theta)=\omega(t, \theta), \quad t \leq 0, \theta \in \mathbb{R}^{+},
\end{gathered}
$$

where $G:(-\infty, 0] \times \mathbb{R}^{+} \rightarrow \mathbb{R}, f_{1},: \mathbb{R}^{+} \times \mathbb{R} \times \mathbb{R}^{+} \rightarrow \mathbb{R}$, $f_{2}: \mathbb{R}^{+} \rightarrow \mathbb{R}$, and $\tau \geq 0$ is a fixed real number.

Remark 9. The linear cases for $f_{1}$ and $f_{2}$ have been considered by many authors. In [8], Piazzera considers the case that $f_{1}: \mathbb{R}^{+} \times \mathbb{R}^{+} \rightarrow \mathbb{R}$ is defined by $f_{1}(t, a)=\mu(a) u(t, a)$, $f_{2} \equiv 0$, and $u(t, 0)=\int_{0}^{\infty} \int_{-r}^{0} \beta(\theta, a) u(t+\theta, a) d \theta d a$, where $\mu \in L_{\text {loc }}^{\infty}\left(\mathbb{R}^{+}, \mathbb{R}^{+}\right)$and $\beta \in L^{\infty}\left([-r, 0] \times \mathbb{R}^{+}, \mathbb{R}^{+}\right)$. In [9], the authors consider the case that $f_{1}(t, a)=\mu(t, a) u(t, a)$ and $u(t, 0)=\int_{0}^{\infty} \beta(t, a) u(t, a) d a$, where $\mu \in L^{\infty}\left(\mathbb{R}^{+} \times \mathbb{R}^{+}, \mathbb{R}^{+}\right)$, $\beta(\cdot, a) \in C^{1}\left(\mathbb{R}^{+}, \mathbb{R}^{+}\right)$, and $\beta(t, \cdot) \in L^{1}\left(\mathbb{R}^{+}, \mathbb{R}^{+}\right)$.

In the first step, we rewrite (53) in operator theoretic form on the Banach space $X$. We define the operator

$$
\mathscr{A} \varphi=-\varphi^{\prime}
$$


with domain $D(\mathscr{A}):=W^{1,1}\left(\mathbb{R}^{+}, \mathbb{R}\right)$. The boundary operator $L: W^{1,1}\left(\mathbb{R}^{+}, \mathbb{R}\right) \rightarrow \mathbb{R}$ is defined by $L \phi=\phi(0)$. Then $A \varphi:=$ $\left(\left.\mathscr{A}\right|_{\operatorname{Ker}(L)}\right) \varphi=-\varphi^{\prime}$ with domain $\left\{\phi \in W^{1,1} \mid \phi(0)=0\right\}$ generates a $C_{0}$-semigroup $T(\cdot)$ on $X$. Before doing the next step, we give the following definition and theorem which characterize the condition $(M)$ in some special cases.

Definition 10. Let $B$ generate a $C_{0}$-semigroup $S(\cdot)$ on a Banach space $\left(Y,\|\cdot\|_{Y}\right)$. The Favard class $\operatorname{Fav}(B)$ is the space $\{x \mid x \in$ $\left.Y, \lim \sup _{t \rightarrow 0^{+}}(1 / t)\|S(t) x-x\|_{Y}<\infty\right\}$.

It is known that $\operatorname{Fav}(B)$ becomes a Banach space if we define $\|x\|_{\operatorname{Fav}(B)}:=\|x\|_{Y}+\lim \sup _{t \rightarrow 0^{+}}(1 / t)\|S(t) x-x\|_{Y}$ for $x \in \operatorname{Fav}(B)$. The following theorem, which gives an important example of operators satisfying condition $(M)$, can be found in $[3,7]$.

Theorem 11. Let $B$ generate a $C_{0}$-semigroup $S(\cdot)$ on a Banach space $Y$. If $C \in B(Y, F a v(B))$, then $C$ satisfies condition ( $M)$ with respect to $S(\cdot)$.

Theorem 12 (see [3]). Let $\lambda>0$. Then $\lambda \in \sigma(A)$ and $P_{\lambda}:=$ $\left(\left.L\right|_{\operatorname{Ker}(\lambda-\mathscr{A})}\right)^{-1}$ is given by $x \mapsto e^{-\lambda \theta} x$ for $x \in \mathbb{R}$ and $\theta \geq 0$. Moreover, $P_{\lambda}$ is a bounded linear operator from $\mathbb{R}$ to $\operatorname{Fav}(A)$.

According to Theorem 12, we know that $\mathscr{A}, X$, and $L$ satisfy assumptions (S1) and (S2). Now, we suppose that $\lambda>0$ in the rest of this section.

For rewriting (53), we define the function

$$
\begin{aligned}
Q_{h}:[0, \infty) & \times \mathscr{P} \longrightarrow X \\
(t, \varphi) & \longmapsto h(t, \varphi) \chi_{[0,1]},
\end{aligned}
$$

where $\chi_{[0,1]}$ denotes the characterization function and $h$ : $[0, \infty) \times \mathscr{P} \rightarrow \mathbb{R}$, and define the linear functional:

$$
\begin{aligned}
& Q: X \longrightarrow \mathbb{R} \\
& \phi \longmapsto \int_{0}^{1} \phi(t) d t .
\end{aligned}
$$

It is easy to see that $Q \in B(X, \mathbb{R})$ and $Q\left(Q_{h}(t, \varphi)\right)=h(t, \varphi)$ for $t \in[0, T]$. Furthermore, by Theorem 12, it follows that $P_{\lambda} Q \epsilon$ $B(X, \operatorname{Fav}(A))$. So, $P_{\lambda} Q$ satisfies condition $(M)$ with respect to $T(\cdot)$ by Theorem 11 . We introduce the following notations:

(i) $u(t)(\cdot):=u(t, \cdot), \varphi(t)(\cdot):=\omega(t, \cdot), v(t)(\cdot):=v(t, \cdot)$, and $C:=P_{\lambda} Q$

(ii) $G_{1}: \mathbb{R}^{+} \times \mathscr{P} \rightarrow X$ is defined by $G_{1}(t, v)(\cdot):=$ $f_{1}\left(t, \int_{0}^{\infty} v(-\tau)(\xi) d \xi, \cdot\right) \quad(v \in \mathscr{P})$; here we assume that $f_{1}\left(t, \int_{0}^{\infty} \psi(a) d a, \cdot\right) \in X$ for each $\psi \in X$ and $t \geq 0$;

(iii) $G_{2}: \mathbb{R}^{+} \times \mathscr{P} \rightarrow \mathbb{R}$ is defined by

$$
G_{2}(t, v):=\int_{0}^{\infty} \int_{-\infty}^{0} G(\theta, a) v(\theta)(a) d \theta d a+f_{2}(t)
$$

$$
(v \in \mathscr{P}),
$$

where $G: \mathbb{R}^{-} \times \mathbb{R}^{+} \rightarrow \mathbb{R}$ and $f_{2}: \mathbb{R}^{+} \rightarrow \mathbb{R}$ are the functions in (53); (iv) $F_{1}(t, v):=Q_{G_{2}}(t, v) \quad(v \quad \in \quad \mathscr{P})$ and $F_{2}(t, v):=$ $\lambda P_{\lambda} G_{2}(t, v)+G_{1}(t, v)$.

Using these notations, we can rewrite (53) as

$$
\begin{gathered}
\frac{d}{d t} u(t)=A\left(u(t)-C F_{1}\left(t, u_{t}\right)\right)+F_{2}\left(t, u_{t}\right), \quad t \in \mathbb{R}^{+}, \\
u_{0}(t)=\varphi(t), \quad t \in \mathbb{R}^{-} .
\end{gathered}
$$

Indeed, condition $u_{0}(t)=\varphi(t), t \in \mathbb{R}^{-}$, means $u(t, \theta)=$ $\omega(t, \theta), t \in \mathbb{R}^{-}, \theta \in \mathbb{R}^{+}$. To see that the differential equation in (58) is equivalent to the first two lines of (53), we first suppose that $u(\cdot)$ is a solution of (58). By the definition of $C$ and the definition of $P_{\lambda}$ in Theorem 12, we see that

$$
\begin{aligned}
\frac{\partial}{\partial t} u(t, \cdot)= & \frac{d}{d t} u(t)=A\left(u(t)-C F_{1}\left(t, u_{t}\right)\right)+F_{2}\left(t, u_{t}\right) \\
= & A\left(u(t)-C F_{1}\left(t, u_{t}\right)\right)+\lambda P_{\lambda} G_{2}\left(t, u_{t}\right)+G_{1}\left(t, u_{t}\right) \\
= & \mathscr{A} u(t)-P_{\lambda} Q Q_{G_{2}}\left(t, u_{t}\right) \\
& +\lambda P_{\lambda} G_{2}\left(t, u_{t}\right)+G_{1}\left(t, u_{t}\right) \\
= & \mathscr{A} u(t)+(\lambda-\mathscr{A}) P_{\lambda} G_{2}\left(t, u_{t}\right)+G_{1}\left(t, u_{t}\right) \\
= & \mathscr{A} u(t)+G_{1}\left(t, u_{t}\right)
\end{aligned}
$$

which is the equation in the first line of (53). Moreover, condition $u(t)-C F_{1}\left(t, u_{t}\right) \in D(A)$ means that

$$
\begin{aligned}
& (u(t))(0)-\left(C F_{1}\left(t, u_{t}\right)\right)(0) \\
& \quad=(u(t))(0)-P_{\lambda} G_{2}\left(t, u_{t}\right)(0) \\
& \quad=(u(t))(0)-e^{-\lambda 0} G_{2}\left(t, u_{t}\right)=0,
\end{aligned}
$$

and so $(u(t))(0)=G_{2}\left(t, u_{t}\right)$, that is, the second line of (53). Hence $(u(\cdot))(\cdot)$ is a solution of (53).

Conversely, it is easy to see that $(u(\cdot))(\cdot)=u(\cdot, \cdot)$ is a solution of (58) wherever $u(\cdot, \cdot)$ is a solution of (53).

In the rest of this section, we suppose that the following conditions on the functions $f_{1}, f_{2}, \omega$, and $G$ hold.

(I) Suppose that a function $f_{1}: \mathbb{R}^{+} \times \mathbb{R} \times \mathbb{R}^{+} \rightarrow \mathbb{R}$ satisfies the following conditions:

(a) For each $(t, s) \in \mathbb{R}^{+} \times \mathbb{R}$, the function $f_{1}(t, s, \cdot) \in$ $X . f_{1}$ is continuously differentiable with respect to the first and second variables.

(b) There are $L>0$ and $\rho \in X$ such that

$$
\begin{aligned}
& \left|f_{1}\left(t, x_{1}, a\right)-f_{1}\left(t, x_{2}, a\right)\right| \\
& \quad+\sum_{i=1}^{2}\left|D_{i} f_{1}\left(t, x_{1}, a\right)-D_{i} f_{1}\left(t, x_{2}, a\right)\right| \\
& \leq L\left|x_{1}-x_{2}\right| \rho(a),
\end{aligned}
$$

for $t \in \mathbb{R}^{+}, a \geq 0$, and $x_{1}, x_{2} \in \mathbb{R}$. 
(II) $f_{2}$ is differentiable. There is a function $g: \mathbb{R}^{+} \rightarrow \mathbb{R}^{+}$ such that $|G(t, a)| \leq g(t)$ for all $a \geq 0$ and $t \leq 0$, and $g(\cdot) e^{-\gamma}$ is integrable on $(-\infty, 0]$.

(a) $\omega \in C^{1}\left(\mathbb{R}^{-} \times \mathbb{R}^{+}, \mathbb{R}\right), t \mapsto \omega(t, \cdot) \in \mathscr{P}$, $t \mapsto(\partial / \partial t) \omega(t, \cdot)$ is continuous in $X$ and $\lim _{t \rightarrow-\infty} e^{\gamma t} \int_{0}^{\infty}|(\partial / \partial t) \omega(t, x)| d x<\infty$.

(b) $\omega$ satisfies

$$
\begin{gathered}
\omega(0,0)=\int_{0}^{\infty} \int_{-\infty}^{0} G(\theta, a) \omega(\theta, a) d \theta d a+f_{2}(0), \\
\frac{\partial}{\partial t} \omega(0, a)=-\frac{\partial}{\partial a} \omega(0, a)+f_{1}\left(0, \int_{0}^{\infty} \omega(-\tau, \xi) d \xi, a\right),
\end{gathered}
$$

for $a \in \mathbb{R}^{+}$.

Now, we are going to verify that all assumptions of Theorem 7 are satisfied.

Lemma 13. $F_{1}$ satisfies hypotheses (H1) and (H2).

Proof. From the definition of $F_{1}$ and assumption (II), it follows that

$$
\begin{aligned}
& \left\|F_{1}\left(t, \psi_{1}\right)-F_{1}\left(t, \psi_{2}\right)\right\| \\
& \quad=\int_{0}^{\infty}\left|G_{2}\left(t, \psi_{1}\right)\left(\chi_{[0,1]}(s)\right)-G_{2}\left(t, \psi_{2}\right)\left(\chi_{[0,1]}(s)\right)\right| d s \\
& \quad=\left|G_{2}\left(t, \psi_{1}\right)-G_{2}\left(t, \psi_{2}\right)\right| \\
& \quad \leq \int_{0}^{\infty} \int_{-\infty}^{0}\left|G(\theta, a) \psi_{1}(\theta)(a)-G(\theta, a) \psi_{2}(\theta)(a)\right| d \theta d a \\
& \leq \int_{-\infty}^{0} \int_{0}^{\infty}\left|G(\theta, a) e^{-\gamma \theta}\right| e^{\gamma \theta}\left|\psi_{1}(\theta)(a)-\psi_{2}(\theta)(a)\right| d a d \theta \\
& \leq \int_{-\infty}^{0} g(\theta) e^{-\gamma \theta} e^{\gamma \theta}\left\|\psi_{1}(\theta)-\psi_{2}(\theta)\right\| d \theta \\
& \quad \leq \int_{-\infty}^{0} g(\theta) e^{-\gamma \theta} d \theta\left\|\psi_{1}-\psi_{2}\right\|_{\mathscr{P}},
\end{aligned}
$$

for $\psi_{1}, \psi_{2} \in \mathscr{P}$. Obviously, $\left\|D_{1} F_{1}\left(t, \psi_{1}\right)-D_{1} F_{1}\left(t, \psi_{2}\right)\right\|=0$. Since

$$
v \longmapsto \int_{0}^{\infty} \int_{-\infty}^{0} G(\theta, a) v(\theta)(a) d \theta d a
$$

is a linear transformation, it follows that

$$
\left\|D_{2} F_{1}\left(t, \psi_{1}\right)-D_{2} F_{1}\left(t, \psi_{2}\right)\right\|=0 .
$$

Consequently, $F_{1}$ satisfies hypotheses (H1) and (H2).

Lemma 14. The function $G_{1}$ satisfies hypotheses (H1) and (H2).
Proof. Suppose that $\phi_{1}, \phi_{2} \in X$ and $t \geq 0$. From assumption (I)(a), it follows that $G_{1}$ is differentiable with respect to the first variable. By assumption (I)(b) and the definition of norm $\|\cdot\|_{\mathscr{P}}$, it follows that

$$
\begin{aligned}
& \left\|G_{1}\left(t, \phi_{1}\right)-G_{1}\left(t, \phi_{2}\right)\right\|+\left\|D_{1} G_{1}\left(t, \phi_{1}\right)-D_{1} G_{1}\left(t, \phi_{2}\right)\right\| \\
& \quad \leq L\left|\int_{0}^{\infty} \phi_{1}(-\tau)(\xi) d \xi-\int_{0}^{\infty} \phi_{2}(-\tau)(\xi) d \xi\right| \times\|\rho\| \\
& \leq L\|\rho\|\left\|\phi_{1}(-\tau)-\phi_{2}(-\tau)\right\| \\
& \quad \leq L e^{\gamma \tau}\|\rho\| \times\left\|\phi_{1}-\phi_{2}\right\|_{\mathscr{P}} .
\end{aligned}
$$

So, hypotheses (H1) and (H2) hold. by

Let $\phi \in \mathscr{P}$ be a fixed element of $\mathscr{P}$. Define $\mathscr{L}_{t, \phi}: \mathscr{P} \rightarrow X$

$$
\mathscr{L}_{t, \phi} \psi:=G_{1}(t, \phi)(\cdot) \int_{0}^{\infty} \psi(-\tau)(\xi) d \xi
$$

for $\psi \in \mathscr{P}$. Obviously, $\mathscr{L}_{t, \phi}$ is well defined and linear. We show that $D_{2} G_{1}(t, \phi)=\mathscr{L}_{t, \phi}$. Let $\psi \in \mathscr{P}$ with $\|\phi-\psi\|_{\mathscr{P}} \neq 0$. Because of assumption (I)((a) and (b)) and the Mean Value Theorem, for each pair $(t, a) \in \mathbb{R}^{+} \times \mathbb{R}^{+}$, there exists $\varsigma_{t, a}$ between $\int_{0}^{\infty} \phi(-\tau)(\xi) d \xi$ and $\int_{0}^{\infty} \psi(-\tau)(\xi) d \xi$ such that

$$
\begin{aligned}
& \frac{\left\|G_{1}(t, \psi)-G_{1}(t, \phi)-\mathscr{L}_{t, \phi}(\psi-\phi)\right\|}{\|\phi-\psi\|_{\mathscr{P}}} \\
& =\frac{1}{\|\phi-\psi\|_{\mathscr{P}}} \\
& \quad \times\left[\| f_{1}\left(t, \int_{0}^{\infty} \phi(-\tau)(\xi) d \xi, a\right)\right. \\
& \quad-f_{1}\left(t, \int_{0}^{\infty} \psi(-\tau)(\xi) d \xi, a\right) \\
& \quad-D_{2} f_{1}\left(t, \int_{0}^{\infty} \phi(-\tau)(\xi) d \xi, a\right) \\
& \left.\quad \times\left(\int_{0}^{\infty} \psi(-\tau)(\xi) d \xi-\int_{0}^{\infty} \phi(-\tau)(\xi) d \xi\right) \|\right] \\
& \quad \times \frac{1}{\|\phi-\psi\|^{\gamma \tau}\|\phi-\psi\|_{\mathscr{P}}}\left[\int_{0}^{\infty} \varsigma_{t, a}-\int_{0}^{\infty} \phi(-\tau)(\xi) d \xi \mid \rho(a) d a\right] \\
& \quad \times\left\|\left[\int_{0}^{\infty} f_{1}\left(t, \varsigma_{t, a}, a\right)-D_{2} f_{1}\left(t, \int_{0}^{\infty} \phi(-\tau)(\xi) d \xi, a\right)\right]\right\| \\
& \quad 1 \xi \int_{0}^{\infty} \psi(-\tau)(\xi) d \xi \mid
\end{aligned}
$$




$$
\begin{aligned}
& \leq L e^{\gamma \tau}\left|\int_{0}^{\infty} \psi(-\tau)(\xi) d \xi-\int_{0}^{\infty} \phi(-\tau)(\xi) d \xi\right| \times\|\rho\| \\
& =L e^{\gamma \tau}\|\psi(-\tau)-\phi(-\tau)\|\|\rho\| \leq L e^{\gamma \tau}\|\psi-\phi\|_{\mathscr{P}}\|\rho\| .
\end{aligned}
$$

Hence $\left\|G_{1}(t, \phi)-G_{1}(t, \psi)-\mathscr{L}_{t, \phi}(\phi-\psi)\right\| /\|\phi-\psi\|_{\mathscr{P}} \rightarrow 0$ as $\|\phi-\psi\|_{\mathscr{P}} \rightarrow 0$. So, we obtain that $D_{2} G_{1}(t, \phi)=\mathscr{L}_{t, \phi}$. Moreover, from assumption (I)(b) and the definition of $\mathscr{P}$, it is easy to see that

$$
\begin{aligned}
& \left\|D_{2} G_{1}\left(t, \phi_{1}\right)-D_{2} G_{1}\left(t, \phi_{2}\right)\right\| \\
& \quad=\left\|\mathscr{L}_{t, \phi_{1},}-\mathscr{L}_{t, \phi_{2}}\right\| \\
& \leq L e^{\gamma \tau}\|\rho\| \times\left\|\phi_{1}-\phi_{2}\right\|_{\mathscr{P}} .
\end{aligned}
$$

Consequently, $G_{1}$ satisfies hypothesis (H2).

Lemma 15. $\mathrm{F}_{2}$ satisfies hypotheses (H1) and (H2).

Proof. In view of Lemma 14, it suffices to show that the operator $\lambda P_{\lambda} G_{2}$ satisfies hypotheses (H1) and (H2). Suppose that $t \geq 0$ and $\psi_{1}, \psi_{2} \in \mathscr{P}$. Using the estimate in the proof of Lemma 13, we see that

$$
\begin{aligned}
& \left\|\lambda P_{\lambda} G_{2}\left(t, \psi_{1}\right)-\lambda P_{\lambda} G_{2}\left(t, \psi_{2}\right)\right\| \\
& \leq\left\|\lambda e^{-\lambda \cdot} G_{2}\left(t, \psi_{1}\right)-\lambda e^{-\lambda \cdot}\left[G_{2}\left(t, \psi_{2}\right)\right]\right\|
\end{aligned}
$$

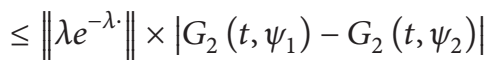

$$
\begin{aligned}
& =\left|G_{2}\left(t, \psi_{1}\right)-G_{2}\left(t, \psi_{2}\right)\right| \\
& \leq \int_{-\infty}^{0} g(\theta) e^{-\gamma \theta} d \theta\left\|\psi_{1}-\psi_{2}\right\|_{\mathscr{P}} .
\end{aligned}
$$

Next, from the definition of $G_{2}$, it is easy to see that

$$
\left\|D_{1}\left(\lambda P_{\lambda} G_{2}\left(t, \psi_{1}\right)\right)-D_{1}\left(\lambda P_{\lambda} G_{2}\left(t, \psi_{2}\right)\right)\right\|=0 .
$$

Since

$$
v \longmapsto \int_{0}^{\infty} \int_{-\infty}^{0} G(\theta, a) v(\theta)(a) d \theta d a
$$

is a linear transformation, it follows that $\| D_{2}\left(\lambda P_{\lambda} G_{2}\left(t, \psi_{1}\right)\right)-$ $D_{2}\left(\lambda P_{\lambda} G_{2}\left(t, \psi_{2}\right)\right) \|=0$. So, $\lambda P_{\lambda} G_{2}$ satisfies hypotheses $(\mathrm{H1})$ and $(\mathrm{H} 2)$. The proof is completed.

Lemma 16. $\varphi \in \mathscr{P}$ is continuously differentiable with $\varphi^{\prime} \in \mathscr{P}$, $\varphi(0)-C F_{1}(0, \varphi) \in D(A)$, and $\varphi^{\prime}(0)=A\left(\varphi(0)-C F_{1}(0, \varphi)\right)+$ $F_{2}(0, \varphi)$.
Proof. Let $t \geq 0$. By the assumption of (III)(a) and the Mean Value Theorem, we know that there is an $\xi_{s}$ between $t$ and $t+h$ such that

$$
\begin{aligned}
& \left\|\frac{\phi(t+h)-\phi(t)}{h}-\phi^{\prime}(t)\right\| \\
& \quad=\int_{0}^{\infty} \frac{|\omega(t+h, s)-\omega(t, s)-(\partial / \partial t) \omega(t, s) h|}{|h|} d s \\
& \quad=\int_{0}^{\infty} \frac{\left|\left[(\partial / \partial t) \omega\left(\xi_{s}, s\right)-(\partial / \partial t) \omega(t, s)\right] h\right|}{|h|} d s \\
& \quad=\int_{0}^{\infty}\left|\frac{\partial}{\partial t} \omega\left(\xi_{s}, s\right)-\frac{\partial}{\partial t} \omega(t, s)\right| d s .
\end{aligned}
$$

The continuity of $t \mapsto(\partial / \partial t) \omega(t, \cdot) d x$ in $X$ implies that the last term goes to 0 as $h \rightarrow 0$. So one can see that $\varphi$ is continuously differentiable in $X$ with $\varphi^{\prime}(t)(\cdot)=(\partial / \partial t) \omega(t, \cdot)$. Moreover, $\phi^{\prime} \in \mathscr{P}$ by assumption (III)(a) and the definition of $\mathscr{P}$. Next, from assumption (III)(b), one can derive that

$$
\begin{aligned}
& \varphi(0)-C F_{1}(0, \varphi) \\
& \quad=\omega(0, s)-e^{-\lambda s}\left[\int_{0}^{\infty} \int_{-\infty}^{0} G(\theta, a) \omega(\theta, a) d \theta d a+f_{2}(0)\right] .
\end{aligned}
$$

Hence, by (III)(a), this implies that $\varphi(0)-C F_{1}(0, \varphi) \in D(A)$. Finally, by using assumption (III)(b) one can derive that

$$
\begin{aligned}
(A( & \left.\left.\varphi(0)-C F_{1}(0, \varphi)\right)+F_{2}(0, \varphi)\right)(s) \\
= & -\frac{\partial}{\partial s}\left[\omega(0, s)-e^{-\lambda s}\left(\int_{0}^{\infty} \int_{-\infty}^{0} G(\theta, a)\right.\right. \\
& \left.\left.\times \omega(\theta, a) d \theta d a+f_{2}(0)\right)\right] \\
& +\lambda e^{-\lambda s}\left[\int_{0}^{\infty} \int_{-\infty}^{0} G(\theta, a) \omega(\theta, a) d \theta d a+f_{2}(0)\right] \\
& +f_{1}\left(0, \int_{0}^{\infty} w(-\tau, \xi) d \xi, s\right) \\
= & -\frac{\partial}{\partial s} \omega(0, s)+f_{1}\left(0, \int_{0}^{\infty} w(-\tau, \xi) d \xi, s\right) \\
= & \frac{\partial}{\partial t} \omega(0, s)=\varphi^{\prime}(0)(s) .
\end{aligned}
$$

The proof is completed.

Consequently, in view of Lemmas 13-16, we can apply Theorem 7 to obtain the following theorem.

Theorem 17. Under assumptions (I)-(III), (58) admits a unique classical solution. 


\section{Solution Semigroups and Regularity}

In this section, the regularity of the mild solution for

$$
\begin{gathered}
\frac{d}{d t} u(t)=A\left(u(t)+C F_{1}\left(u_{t}\right)\right)+F_{2}\left(u_{t}\right), \quad t \geq 0, \\
u_{0}=\varphi \in \mathscr{P}
\end{gathered}
$$

will be found. Throughout this section, we suppose that $F_{1}$ and $F_{2}$ satisfy the following condition:

(H3) $F: \mathscr{P} \rightarrow X$ satisfies a Lipschitz condition; that is, there is a constant $L>0$ such that

$$
\left\|F\left(\psi_{1}\right)-F\left(\psi_{2}\right)\right\| \leq L\left\|\psi_{1}-\psi_{2}\right\|_{\mathscr{P}}
$$

for $\psi_{1}, \psi_{2} \in \mathscr{P}$.

By Theorem 4, we know that (76) has a unique mild solution $u(\cdot, \varphi)$ on $[0, \infty)$ for each $\varphi \in \mathscr{P}$. Hence, we can define the nonlinear operator $U(t)$ on $\mathscr{P}$ by

$$
U(t) \varphi=u_{t}(\cdot, \varphi)=u(t+\cdot, \varphi),
$$

for each $\varphi \in \mathscr{P}$ and $t \geq 0$.

Theorem 18. Under hypotheses $\left(A_{1}\right)$ and $\left(H_{3}\right), U(\cdot)$ is a nonlinear strongly continuous semigroup on $\mathscr{P}$; that is,

(i) $U(0)=I$, where I denotes the identity map,

(ii) $U(t+s)=U(t) U(s)$ for each $t, s \geq 0$,

(iii) $t \mapsto U(t) \varphi$ is a continuous function.

\section{Furthermore,}

(iv) for each $t \geq 0, \varphi \mapsto U(t) \varphi$ is a continuous function,

(v) for each $t \geq 0$ and $\theta \in(-\infty, 0]$, the following translation property holds:

$$
(U(t) \varphi)(\theta)= \begin{cases}(U(t+\theta) \varphi)(0), & t+\theta \geq 0 \\ \varphi(t+\theta), & t+\theta \leq 0\end{cases}
$$

(vi) there exist constants $N_{1}$ and $N_{2}$ such that

$$
\begin{aligned}
& \left\|U(t) \psi_{1}-U(t) \psi_{2}\right\|_{\mathscr{P}} \leq N_{1} e^{N_{2} t}\left\|\psi_{1}-\psi_{2}\right\|_{\mathscr{P}}, \\
& \text { for } \psi_{1}, \psi_{2} \in \mathscr{P} \text { and } t \geq 0 .
\end{aligned}
$$

Proof. (i), (ii), and (v) are easy to see from the definition of $U(\cdot)$. (iii) is obtained from hypothesis (A2) and the definition of $U(t) \varphi$. (iv) follows from (vi). Hence it remains to show (vi). By assumption $(\mathrm{H} 3)$ on $F_{1}$ and $F_{2}$, there is a constant $L>0$ such that

$$
\sum_{i=1}^{2}\left(\left\|F_{i}\left(\psi_{1}\right)-F_{i}\left(\psi_{2}\right)\right\|\right) \leq L\left\|\psi_{1}-\psi_{2}\right\|_{\mathscr{P}},
$$

for $\psi_{1}, \psi_{2} \in \mathscr{P}$. Moreover, we define the following real number:

$$
M_{t}^{\prime \prime}=L M_{t}^{\prime} M e^{|\omega| t}
$$

where $M_{t}^{\prime}=\sup _{0 \leq s \leq t} \max \left\{M_{1}(s), M_{2}(s)\right\}$ and $M_{1}(\cdot)$ and $M_{2}(\cdot)$ are the functions defined in (A1). Let $\psi_{1}, \psi_{2} \in \mathscr{P}$. Use $v_{1}$ and $v_{2}$ to denote $U(\cdot) \psi_{1}$ and $U(\cdot) \psi_{2}$, respectively. By assumption (13) on $C$ and hypotheses (A1) and (H3), it follows that

$$
\begin{aligned}
\| v_{1}(t) & -v_{2}(t) \| \\
\leq & \left\|T(t) \psi_{1}(0)-T(t) \psi_{2}(0)\right\| \\
& +\left\|A \int_{0}^{t} T(t-s) C\left(F_{1}\left(\left(v_{1}\right)_{s}\right)-F_{1}\left(\left(v_{2}\right)_{s}\right)\right) d s\right\| \\
& +\left\|\int_{0}^{t} T(t-s)\left(F_{2}\left(\left(v_{1}\right)_{s}\right)-F_{2}\left(\left(v_{2}\right)_{s}\right)\right) d s\right\| \\
\leq & H M e^{|\omega| t}\left\|\psi_{1}-\psi_{2}\right\|_{\mathscr{P}} \\
& +\sigma_{C}(t) \sup _{0 \leq s \leq t}\left\|F_{1}\left(\left(v_{1}\right)_{s}\right)-F_{1}\left(\left(v_{2}\right)_{s}\right)\right\| \\
& +M e^{|\omega| t} \int_{0}^{t}\left\|F_{2}\left(\left(v_{1}\right)_{s}\right)-F_{2}\left(\left(v_{2}\right)_{s}\right)\right\| d s \\
\leq & H M e^{\omega t}\left\|\psi_{1}-\psi_{2}\right\|_{\mathscr{P}} \\
& +\sigma_{C}(t) L \sup _{0 \leq s \leq t}\left\|\left(v_{1}\right)_{s}-\left(v_{2}\right)_{s}\right\|_{\mathscr{P}} \\
& +\int_{0}^{t} \sup _{0 \leq \zeta \leq s}\left\|\left(v_{1}\right)_{\zeta}-\left(v_{2}\right)_{\zeta}\right\|_{\mathscr{P}} d s,
\end{aligned}
$$

and so

$$
\begin{aligned}
& \left\|\left(v_{1}\right)_{t}-\left(v_{2}\right)_{t}\right\|_{\mathscr{P}} \\
& \leq M_{1}(t) \sup _{0 \leq s \leq t}\left\|v_{1}(s)-v_{2}(s)\right\|+M_{2}(t)\left\|\left(v_{1}\right)_{0}-\left(v_{2}\right)_{0}\right\|_{\mathscr{P}} \\
& \leq M_{1}(t)\left[H M e^{\omega t}\left\|\psi_{1}-\psi_{2}\right\|_{\mathscr{P}}\right. \\
& +\sigma_{C}(t) L \sup _{0 \leq s \leq t}\left\|\left(v_{1}\right)_{s}-\left(v_{2}\right)_{s}\right\|_{\mathscr{P}} \\
& \left.+M e^{|\omega| t} L \int_{0}^{t} \sup _{0 \leq \zeta \leq s}\left\|\left(v_{1}\right)_{\zeta}-\left(v_{2}\right)_{\zeta}\right\|_{\mathscr{P}} d s\right] \\
& +M_{2}(t)\left\|\psi_{1}-\psi_{2}\right\|_{\mathscr{P}} \\
& \leq(H+1) M_{t}^{\prime} M e^{\omega t}\left\|\psi_{1}-\psi_{2}\right\|_{\mathscr{P}} \\
& \quad+\sigma_{C}(t) M_{t}^{\prime} L \sup _{0 \leq s \leq t}\left\|\left(v_{1}\right)_{s}-\left(v_{2}\right)_{s}\right\|_{\mathscr{P}} \\
& +M_{t}^{\prime \prime} \int_{0}^{t} \sup _{0 \leq \zeta} \leq s\left\|\left(v_{1}\right)_{\zeta}-\left(v_{2}\right)_{\zeta}\right\|_{\mathscr{P}} d s
\end{aligned}
$$


for $t \in[0, T]$. We can choose $\varepsilon$ so small that $K:=\sigma_{C}(\varepsilon) L M_{\varepsilon}^{\prime}<$ 1 and it follows that

$$
\begin{aligned}
\sup _{0 \leq s \leq t}\left\|\left(v_{1}\right)_{s}-\left(v_{2}\right)_{s}\right\|_{\mathscr{P}} \\
\leq \frac{1}{1-K}(H+1) M_{\varepsilon}^{\prime} M e^{|\omega| \varepsilon}\left\|\psi_{1}-\psi_{2}\right\|_{\mathscr{P}} \\
\quad+\frac{1}{1-K} M_{\varepsilon}^{\prime \prime} \int_{0}^{t} \sup _{0 \leq \zeta \leq s}\left\|\left(v_{1}\right)_{\zeta}-\left(v_{2}\right)_{\zeta}\right\|_{\mathscr{P}} d s,
\end{aligned}
$$

for $0 \leq t \leq \varepsilon$. By Gronwall's inequality, it follows that

$$
\begin{aligned}
& \sup _{0 \leq s \leq \varepsilon}\left\|U(t) \psi_{1}-U(t) \psi_{2}\right\|_{\mathscr{P}}=\sup _{0 \leq s \leq \varepsilon}\left\|\left(v_{1}\right)_{s}-\left(v_{2}\right)_{s}\right\|_{\mathscr{P}} \\
& \quad \leq K^{\prime}\left\|\psi_{1}-\psi_{2}\right\|_{\mathscr{P}},
\end{aligned}
$$

where

$$
K^{\prime}=\frac{1}{1-K}(H+1) M_{\varepsilon}^{\prime} M e^{|\omega| \varepsilon} e^{\left[(1 /(1-K)) M_{\varepsilon}^{\prime \prime}\right] \varepsilon} .
$$

Since, by (ii) and (86),

$$
\begin{aligned}
\| U & (t) \psi_{1}-U(t) \psi_{2} \|_{\mathscr{P}} \\
& =\left\|U(\varepsilon)\left(U(t-\varepsilon) \psi_{1}\right)-U(\varepsilon)\left(U(t-\varepsilon) \psi_{2}\right)\right\|_{\mathscr{P}} \\
& \leq K^{\prime}\left\|\left(U(t-\varepsilon) \psi_{1}\right)-\left(U(t-\varepsilon) \psi_{2}\right)\right\|_{\mathscr{P}} \\
& \leq\left(K^{\prime}\right)^{n}\left\|\left(U(t-n \varepsilon) \psi_{1}\right)-\left(U(t-n \varepsilon) \psi_{2}\right)\right\|_{\mathscr{P}} \\
& \leq K^{\prime}\left(K^{\prime}\right)^{n}\left\|\psi_{1}-\psi_{2}\right\|_{\mathscr{P}} \\
& =K^{\prime} e^{n \ln K^{\prime}}\left\|\psi_{1}-\psi_{2}\right\|_{\mathscr{P}} \\
& \leq K^{\prime} e^{\omega_{1} t}\left\|\psi_{1}-\psi_{2}\right\|_{\mathscr{P}}
\end{aligned}
$$

for $n \varepsilon \leq t \leq(n+1) \varepsilon$, where $\omega_{1}=\varepsilon^{-1} \ln K^{\prime}$, then $N_{1}:=K^{\prime}$ and $N_{2}:=\omega_{1}$ are desired constants. The proof is completed.

Now, we will focus on the stability near an equilibrium of the nonlinear semigroup $U(\cdot)$ on $\mathscr{P}$. The following assumption is needed.

(H4) $F: \mathscr{P} \rightarrow X$ is continuously Fréchet differentiable with respect to $\left(\mathscr{P},\|\cdot\|_{\mathscr{P}}\right)$ and $F(0)=0$.

Suppose that $F_{1}$ and $F_{2}$ satisfy hypothesis $(\mathrm{H} 4)$ with $F_{1}^{\prime}(0)=$ $L_{1}$ and $F_{2}^{\prime}(0)=L_{2}$ being linear operators on $\mathscr{P}$. Then, by Theorem 4 , the equation

$$
\begin{gathered}
\frac{d}{d t} u(t)=A\left(u(t)+C L_{1}\left(u_{t}\right)\right)+L_{2}\left(u_{t}\right), \quad t \geq 0, \\
u_{0}=\varphi \in \mathscr{P}
\end{gathered}
$$

has a unique mild solution. Let $V(\cdot)$ denote the solution semigroup on $\mathscr{P}$ associated with (89). Then $V(\cdot)$ is a $C_{0}$-semigroup.
Theorem 19. Suppose that $F_{1}$ and $F_{2}$ satisfy hypotheses (H3) and $(H 4)$ with $F_{1}^{\prime}(0)=L_{1}$ and $F_{2}^{\prime}(0)=L_{2}$. Then the Fréchet derivative at 0 of the nonlinear semigroup $U(\cdot)$, associated with (76), is equal to the semigroup $V(\cdot)$ associated with (89).

Proof. First, we show that, for any $T>0, U(t)$ is differentiable with respect to $\mathscr{P}$ for each $t \in[0, T]$. First, since $V(\cdot)$ is a $C_{0^{-}}$ semigroup, there exists a constant $M_{V}$ such that $\|T(t)\| \leq M_{V}$ for $t \in[0, T]$. Fix a $t \in[0, T]$ and let $\varepsilon>0$ be arbitrary. Since the uniqueness of solution and assumption $F(0)=0$ imply $U(t) 0=0$, it is sufficient to find a $\delta>0$ such that

$$
\|U(t) \varphi-V(t) \varphi\|_{\mathscr{P}} \leq \varepsilon\|\varphi\|_{\mathscr{P}}
$$

for each $\|\varphi\|_{\mathscr{P}}<\delta$. By assumptions of $F_{1}$ and $F_{2}$, there is a constant $L>1$ such that

$$
\sum_{i=1}^{2}\left(\left\|F_{i}\left(\phi_{1}\right)-F_{i}\left(\phi_{2}\right)\right\|\right) \leq L\left\|\phi_{1}-\phi_{2}\right\|_{\mathscr{P}}
$$

for $\phi_{1}, \phi_{2} \in \mathscr{P}$. Moreover, we define the following real number:

$$
M_{t}^{\prime \prime}=L M_{1}^{\prime} M e^{|\omega| t}
$$

where $M_{1}^{\prime}=\sup _{0 \leq s \leq T} M_{1}(\cdot)$ and $M_{1}(\cdot)$ is the function defined in (A1). Let $u$ and $v$ denote the mild solutions of (76) and (89), respectively. Let $r>0$. For all $0 \leq \tau \leq t \leq r$, we have

$$
\begin{aligned}
& \|U(\tau) \varphi-V(\tau) \varphi\|_{\mathscr{P}} \\
& \leq M_{1}(\tau) \sup _{0 \leq s \leq \tau}\|u(s)-v(s)\| \leq M_{1}^{\prime} \sup _{0 \leq s \leq t}\|u(s)-v(s)\| \\
& \leq M_{1}^{\prime} \sup _{0 \leq s \leq t}\left\{\| A \int_{0}^{s} T(t-\varsigma)\right. \\
& +\quad \times C\left[F_{1}(U(\varsigma) \varphi)-L_{1}(V(\varsigma) \varphi)\right] d \varsigma\|\| \int_{0}^{s} T(t-\varsigma) \\
& \left.\quad \times\left(F_{2}(U(\varsigma) \varphi)-L_{2}(V(\varsigma) \varphi)\right) d \varsigma \|\right\} \\
& +M_{1}^{\prime} \sigma_{C}(t) \sup _{0 \leq s \leq t}\left\|F_{1}(U(s) \varphi)-L_{1}(V(s) \varphi)\right\| \\
& +M_{1}^{\prime} M e^{|\omega| t} \int_{0}^{t}\left\|F_{2}(U(s) \varphi)-L_{2}(V(s) \varphi)\right\| d s \\
& +M_{1}^{\prime} M e^{|\omega| t}\left(\int_{0}^{t}\left\|F_{2}(U(s) \varphi)-F_{2}(V(s) \varphi)\right\| d s\right. \\
& +\sup _{0 \leq s \leq t}\left\{\left\|F_{1}(U(s) \varphi)-F_{1}(V(s) \varphi)\right\|\right. \\
& \left.+\left\|F_{1}(V(s) \varphi)-L_{1}(V(s) \varphi)\right\|\right\}
\end{aligned}
$$




$$
\begin{aligned}
\leq & M_{r}^{\prime \prime}\left(\sigma_{C}(r)+r\right) \sup _{0 \leq s \leq t}\|U(s) \varphi-V(s) \varphi\|_{\mathscr{P}} \\
+ & M_{r}^{\prime \prime}\left[\sigma_{C}(r) \sup _{0 \leq s \leq t}\left\|F_{1}(V(s) \varphi)-L_{1}(V(s) \varphi)\right\|\right. \\
& \left.\quad+\int_{0}^{t} \sup _{0 \leq \eta \leq s}\left\|F_{2}(V(\eta) \varphi)-L_{2}(V(\eta) \varphi)\right\| d s\right] .
\end{aligned}
$$

We can choose $r$ so small that $K:=M_{r}^{\prime \prime}\left(\sigma_{C}(r)+r\right)<1$. Hence, the last inequality implies that

$$
\begin{aligned}
\sup _{0 \leq s \leq t}\|U(s) \varphi-V(s) \varphi\|_{\mathscr{P}} \\
\leq \frac{M_{r}^{\prime \prime}}{1-K} \sigma_{C}(r) \sup _{0 \leq s \leq t}\left\|F_{1}(V(s) \varphi)-L_{1}(V(s) \varphi)\right\| \\
\quad+\frac{M_{r}^{\prime \prime}}{1-K} \int_{0}^{t} \sup _{0 \leq \eta \leq s}\left\|F_{2}(V(\eta) \varphi)-L_{2}(V(\eta) \varphi)\right\| d s,
\end{aligned}
$$

for $t \in[0, r]$.

Since $F_{1}$ is differentiable at 0 and $F_{1}(0)=0$ with Fréchet derivative $L_{1}$, from the definition of Fréchet derivative and linearity of $V(s)$, for each $\varepsilon^{\prime}>0$, there exists a $\delta>0$ such that

$$
\begin{aligned}
& \left\|F_{1}(V(s) \varphi)-L_{1}(V(s) \varphi)\right\| \\
& \quad=\left\|F_{1}(V(s) \varphi)-F_{1}(V(s) 0)-L_{1}((V(s) \varphi-V(s) 0))\right\| \\
& \quad \leq \varepsilon^{\prime}\|V(s) \varphi-V(s) 0\|_{\mathscr{P}}=\varepsilon^{\prime} M_{V}\|\varphi\|_{\mathscr{P}},
\end{aligned}
$$

for $\|\varphi\|_{\mathscr{P}}<\delta$ and $s \in[0, t]$, and so

$$
\sup _{0 \leq s \leq t}\left\|F_{1}(V(s) \varphi)-L_{1}(V(s) \varphi)\right\| \leq \varepsilon^{\prime} M_{V}\|\varphi\|_{\mathscr{P}}
$$

for $t \in[0, r]$. Let $\varepsilon^{\prime}=\left(2 M_{r}^{\prime \prime}\right)^{-1}\left(1 /\left(1+\sigma_{C}(r)\right)\right)\left((1-K) \varepsilon / M_{V}\right)$. Then for given $\varepsilon$ there exists a $\delta>0$ such that

$$
\begin{aligned}
& \sup _{0 \leq s \leq t}\left\|F_{1}(V(s) \varphi)-L_{1}(V(s) \varphi)\right\| \\
& \quad \leq\left(2 M_{r}^{\prime \prime}\right)^{-1} \frac{1}{1+\sigma_{C}(r)}(1-K) \varepsilon\|\varphi\|_{\mathscr{P}}
\end{aligned}
$$

for $\|\varphi\|_{\mathscr{P}}<\delta$ and $t \in[0, r]$. Similarly,

$$
\begin{aligned}
& \int_{0}^{t} \sup _{0 \leq \eta \leq s}\left\|F_{2}(V(\eta) \varphi)-L_{2}(V(\eta) \varphi)\right\| d s \\
& \quad \leq\left(2 M_{r}^{\prime \prime}\right)^{-1}(1-K) \varepsilon\|\varphi\|_{\mathscr{P}},
\end{aligned}
$$

for $\|\varphi\|_{\mathscr{P}}<\delta$ and $t \in[0, r]$. Consequently, $\|U(t) \varphi-V(t) \varphi\|_{\mathscr{P}}$ $\leq \varepsilon\|\varphi\|_{\mathscr{P}}$ for $\|\varphi\|_{\mathscr{P}}<\delta$ and $t \in[0, r]$; that is, $\varphi \mapsto U(t) \varphi$ is Fréchet differentiable for $t \in[0, r]$.
If $r \leq t=r+h \leq 2 r$, then, by (ii) and (iv) of Theorem 18, it follows that

$$
\begin{aligned}
&\|U(t) \varphi-V(t) \varphi\|_{\mathscr{P}} \\
&= \| U(r) U(h) \varphi-U(r) V(h) \varphi \\
&+U(r) V(h) \varphi-V(r) V(h) \varphi \|_{\mathscr{P}} \\
& \leq\|U(r) U(h) \varphi-U(r) V(h) \varphi\|_{\mathscr{P}} \\
&+\|U(r) V(h) \varphi-V(r) V(h) \varphi\|_{\mathscr{P}} \\
& \leq N_{1} e^{N_{2} r}\|U(h) \varphi-V(h) \varphi\|_{\mathscr{P}}+\varepsilon\|V(h) \varphi\|_{\mathscr{P}} \\
& \leq N_{1} e^{N_{2} r} \varepsilon\|\varphi\|_{\mathscr{P}}+\varepsilon M_{V}\|\varphi\|_{\mathscr{P}}
\end{aligned}
$$

for $\varphi \in \mathscr{P}$ with $\|\varphi\|_{\mathscr{P}}<\delta$. Thus, for given $\varepsilon_{1}>0$, letting $\varepsilon=\left(N_{1} e^{N_{2} r}+M_{V}\right)^{-1} \varepsilon_{1}$, we have shown that

$$
\|U(t) \varphi-V(t) \varphi\|_{\mathscr{P}} \leq \varepsilon_{1}\|\varphi\|_{\mathscr{P}}
$$

for $\|\varphi\|_{\mathscr{P}}<\delta$. So, the mapping $\varphi \mapsto U(t) \varphi$ is Fréchet differentiable for $t \in[0,2 r]$ and the Fréchet derivative equals the map $\varphi \mapsto V(t) \varphi$. Repeating this argument, we can get the conclusion.

Definition 20 (see [19]). Let $U(\cdot)$ be a strongly continuous semigroup on a Banach space $\left(W,\|\cdot\|_{W}\right)$. A point $w \in W$ is called an equilibrium of $U(\cdot)$ if $U(t) w=w$ for all $t \geq 0$. An equilibrium $w \in W$ is said to be exponentially stable if there exist $\delta>0, v>0$, and $N>1$ such that

$$
\|U(t) x-w\|_{W} \leq N e^{-v t}\|x-w\|_{W},
$$

for $t \geq 0$ and $\|x-w\|_{W} \leq \delta$.

When $U(\cdot)$ is a linear semigroup, this definition reduces to the usual definition of exponential stability of $C_{0}$-semigroups: $\|U(t)\| \leq N e^{-\vartheta t}$.

Theorem 21 (see [19]). Let $U(\cdot)$ be a nonlinear strongly continuous semigroup in a Banach space $W$. Assume that $w \in W$ is an equilibrium of $U(\cdot)$ such that $U(t)$ is Fréchet differentiable at $w$ for each $t \geq 0$, with $V(t)$ the Fréchet derivative at $w$ of $U(t)$. Then, $V(\cdot)$ is a strongly continuous semigroup of bounded linear operators on $W$. Moreover, if $V(\cdot)$ is exponentially stable, then $w$ is an exponentially stable equilibrium of $U(\cdot)$.

Since (H4) implies that 0 is an equilibrium of the semigroup $U(\cdot)$ in Theorem 19, by Theorem 21, we have the following consequence.

Theorem 22. Suppose that $F_{1}$ and $F_{2}$ satisfy hypotheses (H3) and (H4). If $V(\cdot)$ is exponentially stable on $\mathscr{P}$, that is, there exist constants $\beta \leq 1$ and $\omega_{1}>0$ such that $\|V(t) \varphi\|_{\mathscr{P}} \leq \beta e^{-\omega_{1} t}\|\varphi\|_{\mathscr{P}}$ for $t \geq 0$ and $\varphi \in \mathscr{P}$, then zero is an exponentially stable equilibrium of $U(\cdot)$ on $\mathscr{P}$; that is, there exist $\delta>0, v>0$, and $N>1$ such that

$$
\|U(t) \varphi\|_{\mathscr{P}} \leq N e^{-v t}\|\varphi\|_{\mathscr{P}}
$$

for $t \geq 0$ and $\|\varphi\|_{\mathscr{P}} \leq \delta$. 
Remark 23. Theorems 19 and 22 for the special case that $C=$ 0 and $A$ satisfies the Hille-Yosida condition can be found in [10].

\section{Conflict of Interests}

The author declares that there is no conflict of interests regarding the publication of this paper.

\section{References}

[1] The Dynamics of Physiologically Structured Populations, vol. 68 of Lecture Notes in Biomathematics, Springer, Berlin, Germany, 1986, edited by J. A. J. Metz and O. Diekman.

[2] G. F. Webb, Theory of Nonlinear Age-Dependent Population Dynamics, vol. 89, Marcel Dekker, New York, NY, USA, 1985.

[3] W. Desch and W. Schappacher, "Some generation results for perturbed semigroups," in Semigroup Theory and Applications (Trieste, 1987), vol. 116 of Lecture Notes in Pure and Applied Mathematics, pp. 125-152, Dekker, New York, NY, USA, 1989.

[4] W. Desch, W. Schappacher, and K. P. Zhang, "Semilinear evolution equations," Houston Journal of Mathematics, vol. 15, no. 4, pp. 527-552, 1989.

[5] H. O. Fattorini, "Boundary control systems," SIAM Journal on Control and Optimization, vol. 6, pp. 213-229, 1968.

[6] K.-J. Engel and R. Nagel, One-Parameter Semigroups for Linear Evolution Equations, vol. 194 of Graduate Texts in Mathematics, Springer, New York, NY, USA, 2000.

[7] S. Piskarëv and S.-Y. Shaw, "Multiplicative perturbations of $C_{0^{-}}$ semigroups and some applications to step responses and cumulative outputs," Journal of Functional Analysis, vol. 128, no. 2, pp. 315-340, 1995.

[8] S. Piazzera, "An age-dependent population equation with delayed birth process," Mathematical Methods in the Applied Sciences, vol. 27, no. 4, pp. 427-439, 2004.

[9] S. Boulite, L. Maniar, and M. Moussi, "Wellposedness and asymptotic behaviour of non-autonomous boundary Cauchy problems," Forum Mathematicum, vol. 18, no. 4, pp. 611-638, 2006.

[10] M. Adimy, H. Bouzahir, and K. Ezzinbi, "Local existence and stability for some partial functional differential equations with infinite delay," Nonlinear Analysis: Theory, Methods \& Applications, vol. 48, no. 3, pp. 323-348, 2002.

[11] M. Adimy and K. Ezzinbi, "A class of linear partial neutral functional-differential equations with nondense domain," Journal of Differential Equations, vol. 147, no. 2, pp. 285-332, 1998.

[12] J.-C. Chang, "Solutions to non-autonomous integrodifferential equations with infinite delay," Journal of Mathematical Analysis and Applications, vol. 331, no. 1, pp. 137-151, 2007.

[13] Y. Hino, S. Murakami, and T. Naito, Functional-Differential Equations with Infinite Delay, vol. 1473 of Lecture Notes in Mathematics, Springer, Berlin, Germany, 1991.

[14] H. R. Henríquez, "Regularity of solutions of abstract retarded functional-differential equations with unbounded delay," Nonlinear Analysis: Theory, Methods \& Applications, vol. 28, no. 3, pp. 513-531, 1997.

[15] H. R. Henríquez, "Periodic solutions of quasi-linear partial functional-differential equations with unbounded delay," Funkcialaj Ekvacioj, vol. 37, no. 2, pp. 329-343, 1994.
[16] H. R. Henríquez, "Approximation of abstract functional-differential equations with unbounded delay," Indian Journal of Pure and Applied Mathematics, vol. 27, no. 4, pp. 357-386, 1996.

[17] J. K. Hale and J. Kato, "Phase space for retarded equations with infinite delay," Funkcialaj Ekvacioj, vol. 21, no. 1, pp. 11-41, 1978.

[18] H. Bouzahir, Contribution à l'étude des aspects quantitatif et qualitatif pour une classe d'équations différentielles à retard infini, en dimension infinie [Doctorial disseratation], Cadi Ayyad University, Marrakech, Morocco, 2001.

[19] W. Desch and W. Schappacher, "Linearized stability for nonlinear semigroups," in Differential Equations in Banach Spaces, A. Favini and E. Obrecht, Eds., vol. 1223 of Lecture Notes in Mathematics, pp. 61-73, Springer, Berlin, 1986. 


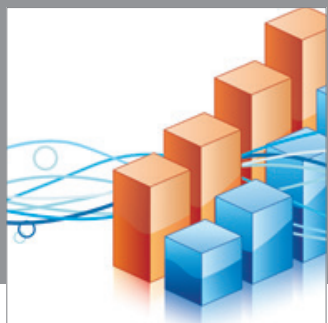

Advances in

Operations Research

mansans

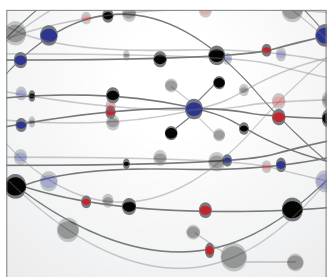

The Scientific World Journal
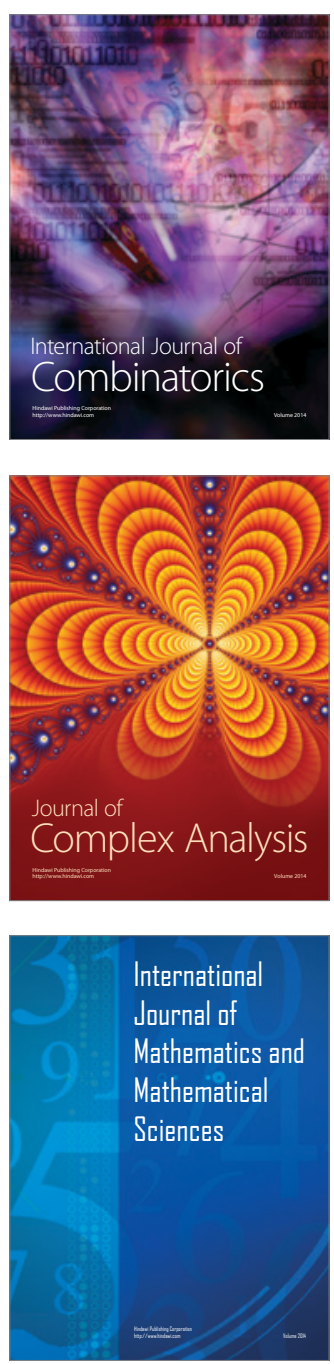
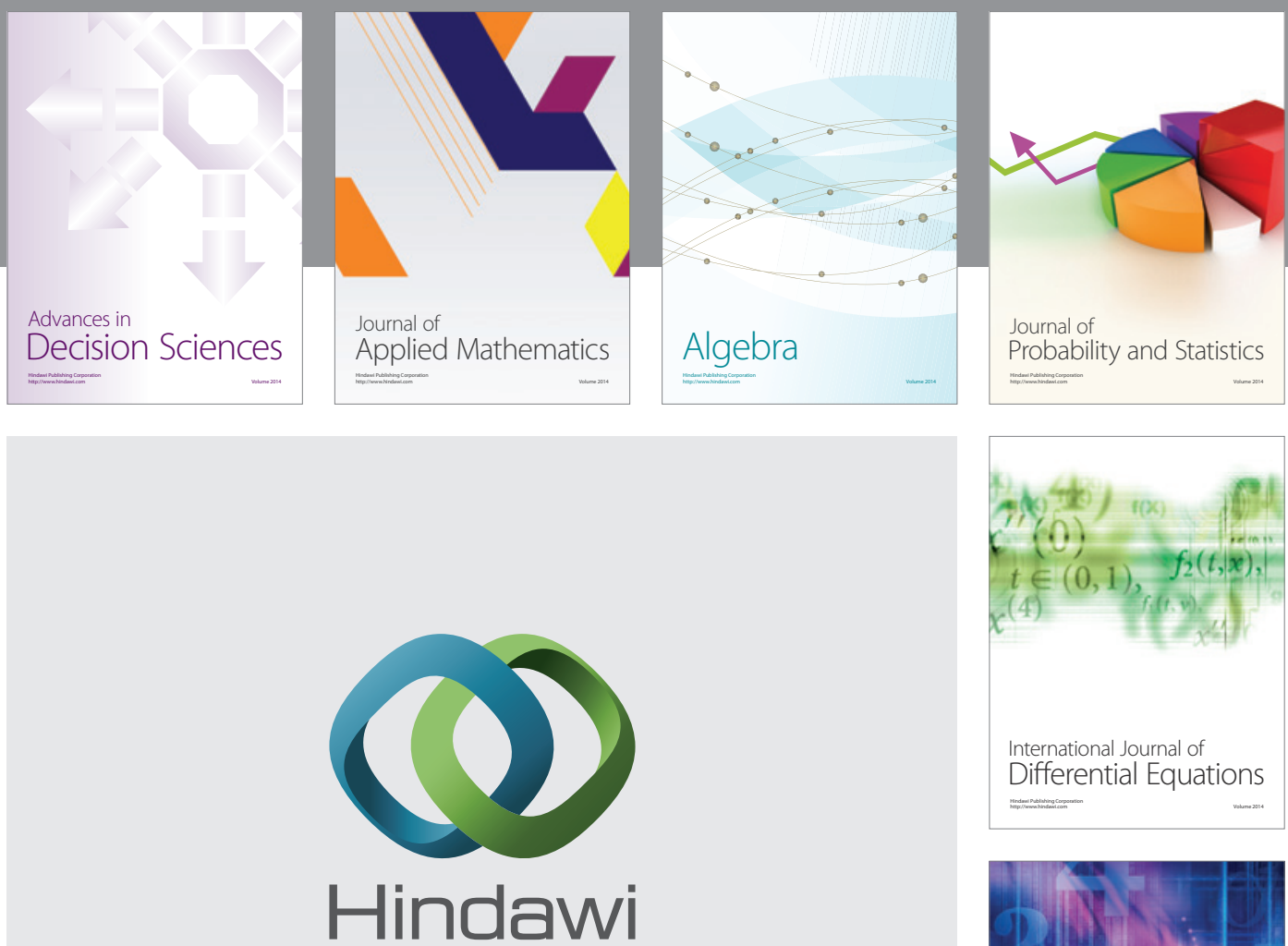

Submit your manuscripts at http://www.hindawi.com
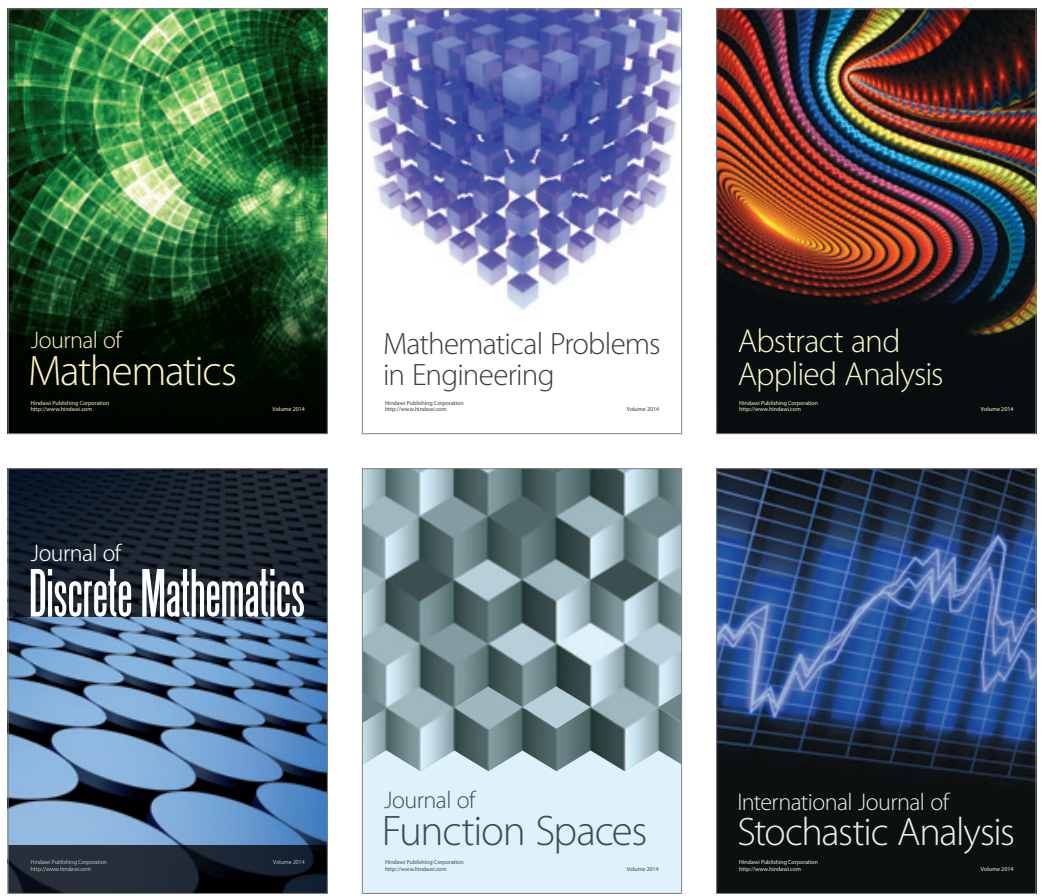

Journal of

Function Spaces

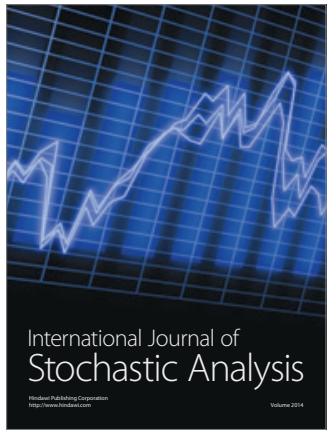

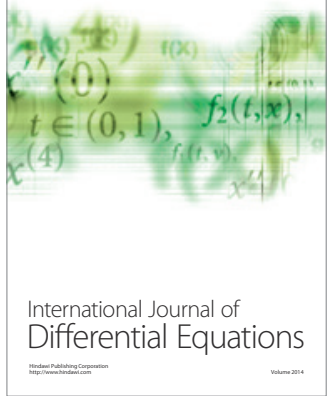
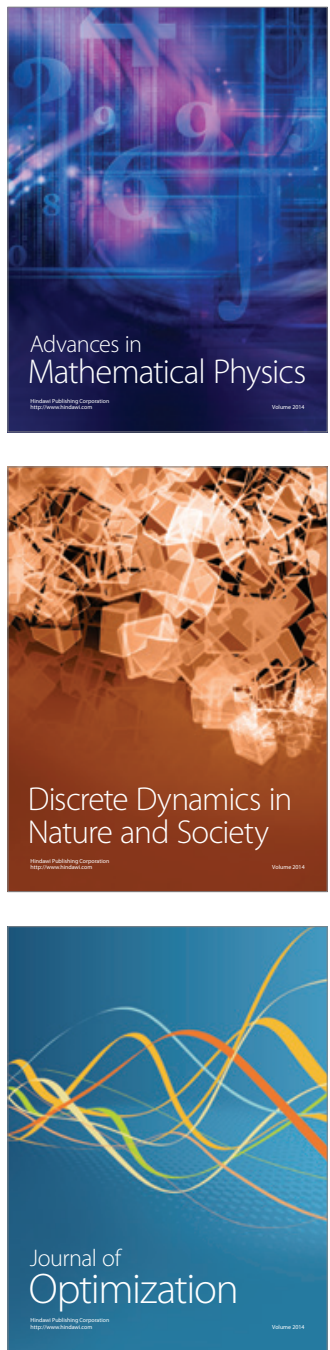